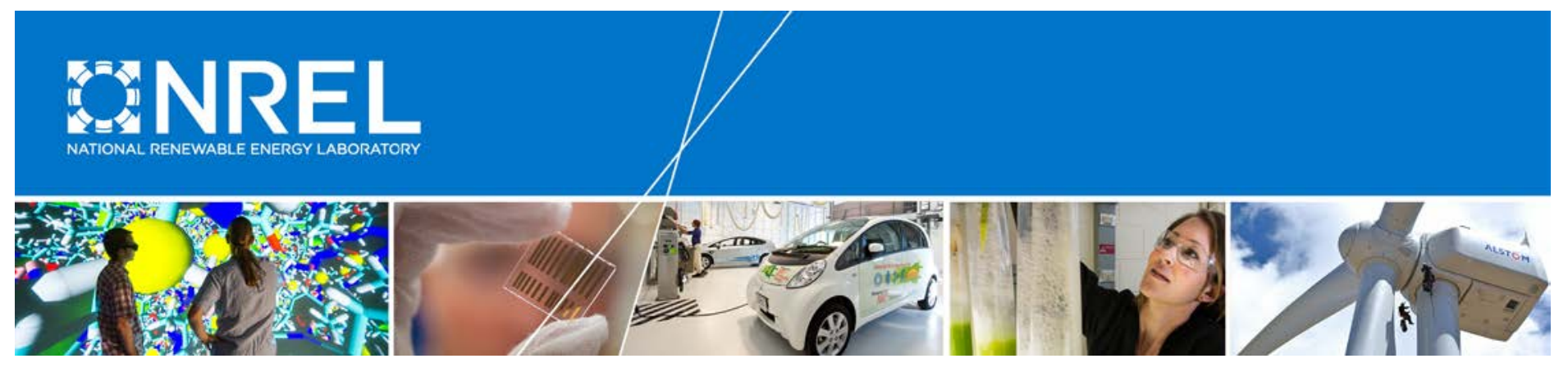

\title{
On the Inclusion of Energy- Shifting Demand Response in Production Cost Models: Methodology and a Case Study
}

Niamh O'Connell

Technical University of Denmark

Elaine Hale, Ian Doebber, and Jennie Jorgenson National Renewable Energy Laboratory

NREL is a national laboratory of the U.S. Department of Energy Office of Energy Efficiency \& Renewable Energy Operated by the Alliance for Sustainable Energy, LLC

This report is available at no cost from the National Renewable Energy Laboratory (NREL) at www.nrel.gov/publications.

Technical Report

NREL/TP-6A20-64465

July 2015

Contract No. DE-AC36-08GO28308 


\section{On the Inclusion of Energy- Shifting Demand Response in Production Cost Models: Methodology and a Case Study}

Niamh O'Connell

Technical University of Denmark

Elaine Hale, Ian Doebber, and Jennie Jorgenson National Renewable Energy Laboratory

Prepared under Task No. 066A.1502

NREL is a national laboratory of the U.S. Department of Energy Office of Energy Efficiency \& Renewable Energy Operated by the Alliance for Sustainable Energy, LLC

This report is available at no cost from the National Renewable Energy Laboratory (NREL) at www.nrel.gov/publications.

National Renewable Energy Laboratory 15013 Denver West Parkway Golden, CO 80401

303-275-3000 • www.nrel.gov
Technical Report

NREL/TP-6A20-64465

July 2015

Contract No. DE-AC36-08GO28308 


\section{NOTICE}

This report was prepared as an account of work sponsored by an agency of the United States government. Neither the United States government nor any agency thereof, nor any of their employees, makes any warranty, express or implied, or assumes any legal liability or responsibility for the accuracy, completeness, or usefulness of any information, apparatus, product, or process disclosed, or represents that its use would not infringe privately owned rights. Reference herein to any specific commercial product, process, or service by trade name, trademark, manufacturer, or otherwise does not necessarily constitute or imply its endorsement, recommendation, or favoring by the United States government or any agency thereof. The views and opinions of authors expressed herein do not necessarily state or reflect those of the United States government or any agency thereof.

This report is available at no cost from the National Renewable Energy Laboratory (NREL) at www.nrel.gov/publications.

Available electronically at SciTech Connect http:/www.osti.gov/scitech

Available for a processing fee to U.S. Department of Energy and its contractors, in paper, from:

U.S. Department of Energy

Office of Scientific and Technical Information

P.O. Box 62

Oak Ridge, TN 37831-0062

OSTI http://www.osti.gov

Phone: 865.576 .8401

Fax: 865.576.5728

Email: reports@osti.gov

Available for sale to the public, in paper, from:

U.S. Department of Commerce

National Technical Information Service

5301 Shawnee Road

Alexandria, VA 22312

NTIS http://www.ntis.gov

Phone: 800.553 .6847 or 703.605 .6000

Fax: 703.605.6900

Email: orders@ntis.gov 


\section{Acknowledgments}

The authors would like to thank the following individuals for their valuable input and comments during the analysis and publication process: Giulia Gallo, Noel Merket, Daniel Macumber, Jordan Clark, Adam Hirsch, Paul Denholm, Ben Kroposki, Aaron Bloom, Pierre Pinson, Henrik Madsen, and Mark O’Malley. Any errors or omissions are solely the responsibility of the authors. 


\section{Executive Summary}

In the context of future power system requirements for additional flexibility, demand response (DR) is an attractive potential resource. Its proponents widely laud its prospective benefits, which include enabling higher penetrations of variable renewable generation at lower cost than alternative storage technologies, and improving economic efficiency. In practice, DR from the commercial and residential sectors is largely an emerging, not a mature, resource, and its actual costs and benefits need to be studied to determine promising combinations of physical DR resource, enabling controls and communications, power system characteristics, regulatory environments, market structures, and business models. The work described in this report focuses on the enablement of such analysis from the production cost modeling perspective. In particular, we contribute a bottom-up methodology for modeling load-shifting DR in production cost models. The resulting model is sufficiently detailed to reflect the physical characteristics and constraints of the underlying flexible load, and includes the possibility of capturing diurnal and seasonal variations in the resource. Nonetheless, the model is of low complexity and thus suitable for inclusion in conventional unit commitment and market clearing algorithms. The ability to simulate DR as an operational resource on a power system over a year facilitates an assessment of its time-varying value to the power system.

The modeling methodology is demonstrated through a case study of aggregated supermarket refrigeration systems providing balancing energy reserves in real-time markets at different levels of variable generation (VG). This DR resource is implemented in a test power system that represents a subset of the U.S Western Interconnection centered on Colorado. The value of DR from the population of supermarkets in Colorado is found to be $\$ 32.85$ per kilowatt-year (kW-yr) presuming no other DR resources. The value decreases significantly (to $\$ 6.95 / \mathrm{kW}$-year in the most extreme case) when we increase the capacity of the DR resource to naïvely represent the incorporation of DR from other flexible loads (in actuality, other DR resources will have different characteristics, such that the decrease in value will not be as steep). Refrigeration DR is found to offer greater value to the power system during the winter months than the summer months due to operational constraints that limit the flexibility of the resource during the summer. The value of DR is found to increase as the penetration of VG increases, reaching $\$ 46.05 / \mathrm{kW}$ year for our baseline DR penetration and a variable generation (VG) penetration of $55 \%$. We do see a plateau in the value of DR going from $45 \%$ to $55 \% \mathrm{VG}$. This is attributable to the inability of DR to provide energy storage on horizons longer than 24 hours.

Overall, this work is a study in methodology. The case study is included primarily to show that the model is working properly and that this line of research is worthwhile. The reported numbers do not represent a true value of DR, but they do suggest orders of magnitude for a particular DR resource providing a particular grid service in a particular power system; they also confirm expected correlation directions between value and DR penetration (decreasing) and between value and VG penetration (increasing). Future work includes extending this method and developing new methods to be able to model physically realistic DR resources at scale. Some important aspects not studied here include capturing all possible value streams for a single resource (capacity, energy, and ancillary service values), simultaneously evaluating DR from multiple resources, and economically competing DR resources based on their costs of enablement and the trade-offs between end-user disutility and participation payments. 


\section{Table of Contents}

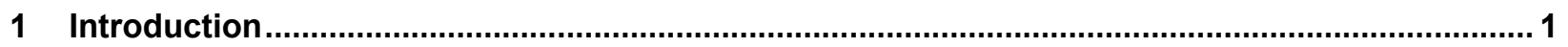

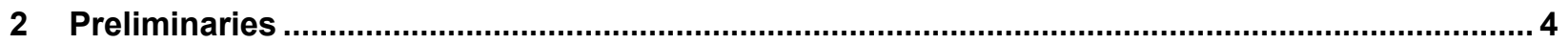

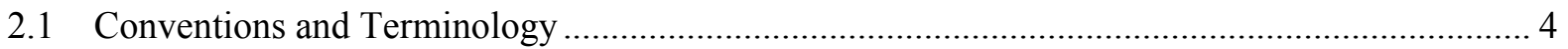

2.2 Running Example: Supermarket Refrigeration ........................................................... 4

3 Characterizing Thermal-Electric Load-Shifting Demand Response for Power System Studies.. 5

3.1 Characterizing Demand Response through the Saturation Curve .............................................. 5

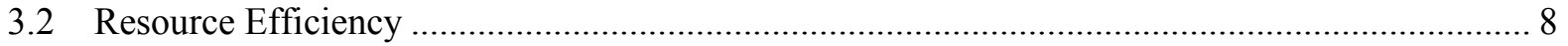

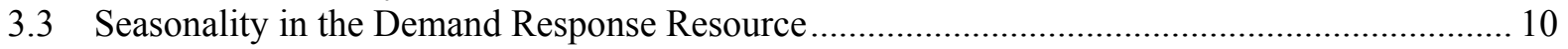

4 Mathematical Representation of Demand Response Resource ............................................. 13

4.1 Theoretically Optimal Formulation ..................................................................................... 13

4.2 Practically Implementable Formulation Suitable for PLEXOS …......................................... 16

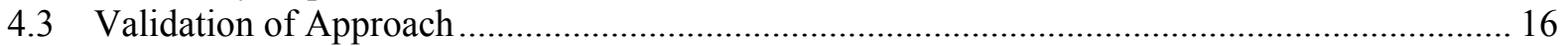

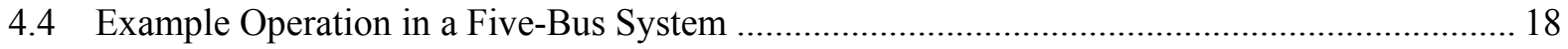

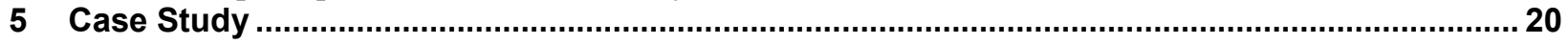

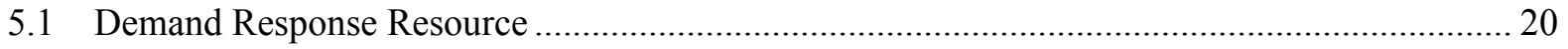

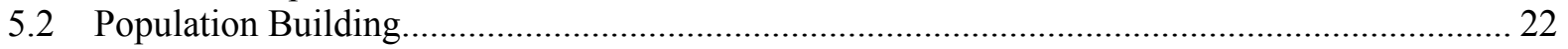

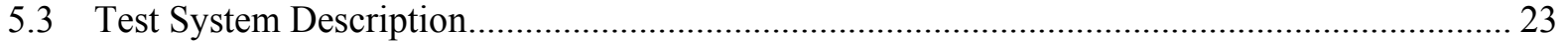

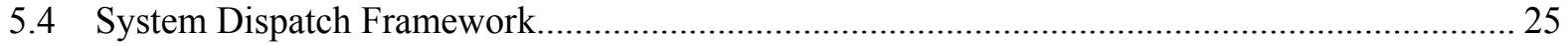

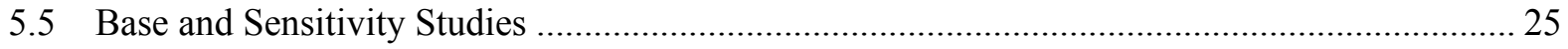

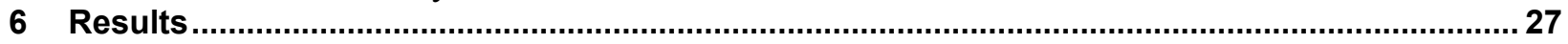

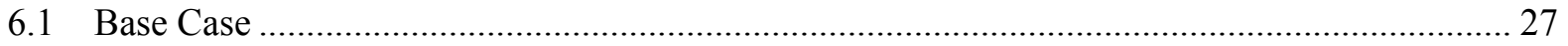

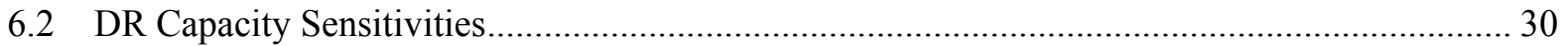

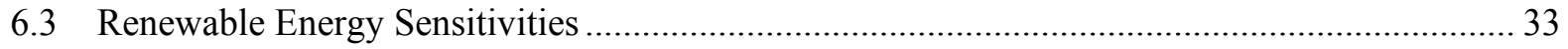

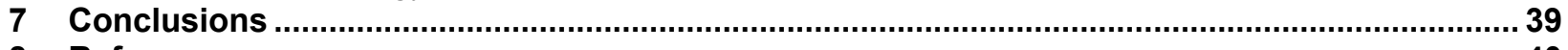

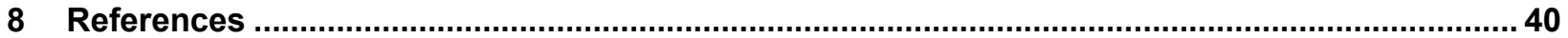




\section{List of Figures}

Figure 1. Power consumption in refrigeration system when required to follow a power

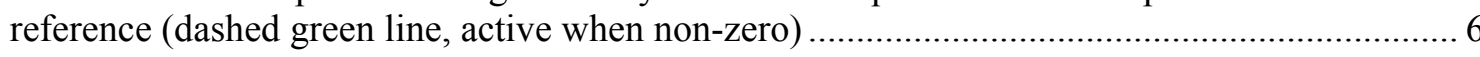

Figure 2. Temperature in the refrigeration system, subject to upper and lower limits

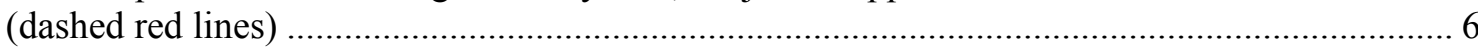

Figure 3. Saturation curve of a sample refrigeration system as seen from the power

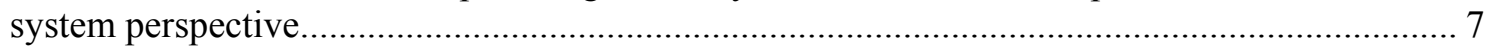

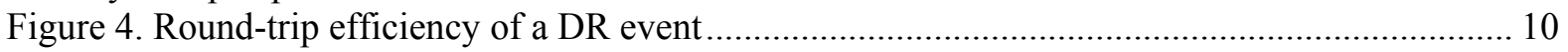

Figure 5. Normalized consumption of electrical end uses suitable for load shifting........................... 11

Figure 6. Variations in available flexibility at the device level, using the example of

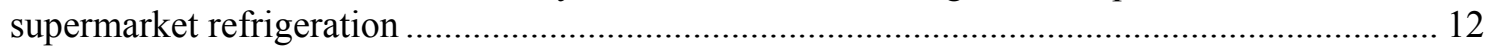

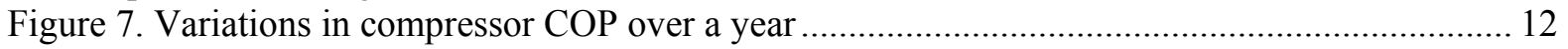

Figure 8. DR resource behaviour over a one-week period as simulated on a five-bus power

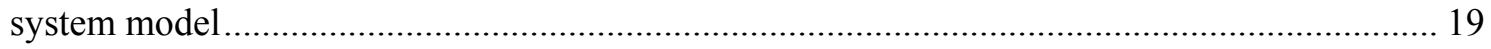

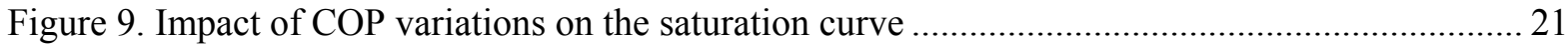

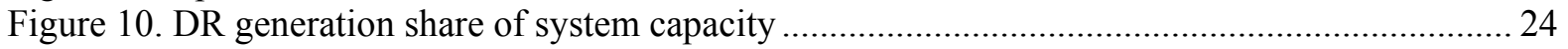

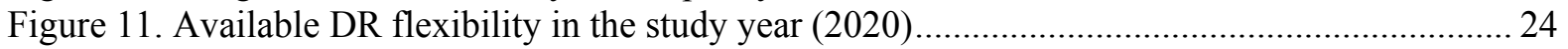

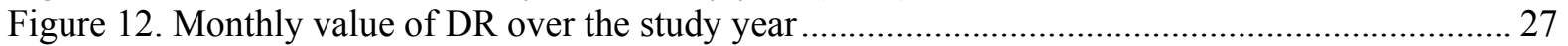

Figure 13. Monthly avoided curtailment of renewables achieved through the implementation

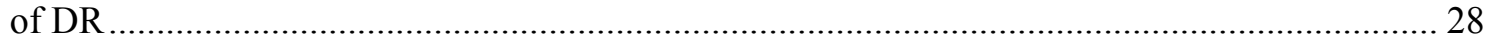

Figure 14. Revenue breakdown by DR resource definition offered for dispatch ...............................29

Figure 15. Monthly net revenue of the entire DR resource over the study year.................................29

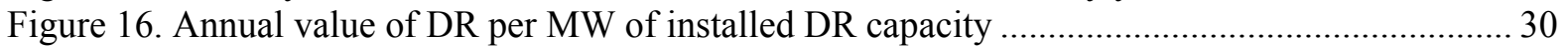

Figure 17. Annual DR revenue per MW of installed DR capacity ..................................................... 31

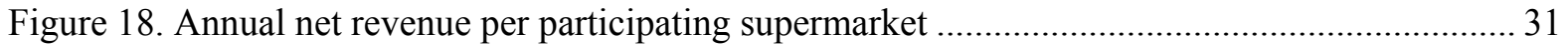

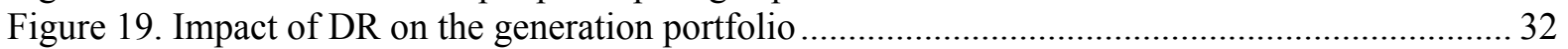

Figure 20. Price duration curves at different levels of installed DR capacity ................................... 33

Figure 21. Annual value of DR per MW of installed capacity with increasing penetrations

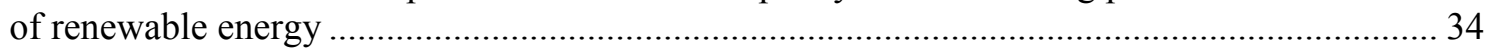

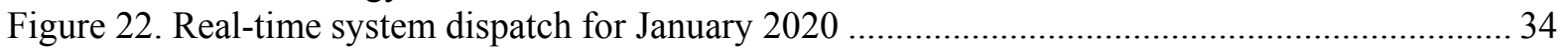

Figure 23. Curtailed renewable generation during January 2020 ................................................... 35

Figure 24. Monthly DR load reduction for each renewable energy penetration scenario .................... 35

Figure 25 Impact of DR on Generation with increasing penetrations of renewable generation

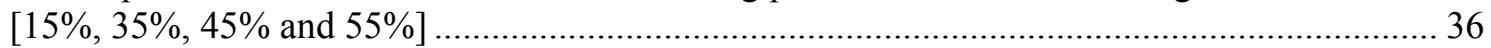

Figure 26. Annual avoided curtailment of renewable generation per MW of enabled DR capacity

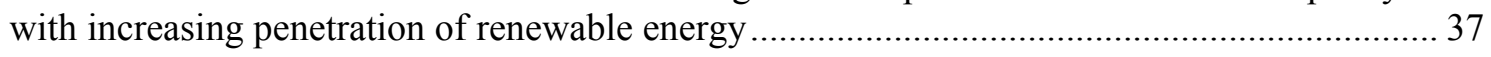

Figure 27. Annual value of DR per participating supermarket with increasing penetrations of

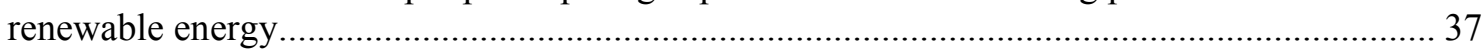

Figure 28. Annual net revenue per supermarket with increasing penetrations of renewable

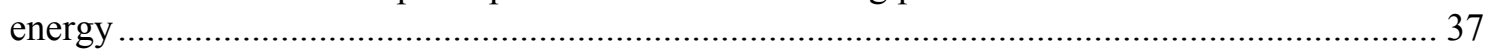

Figure 29. Price duration curve with increasing penetration of renewable energy and the base

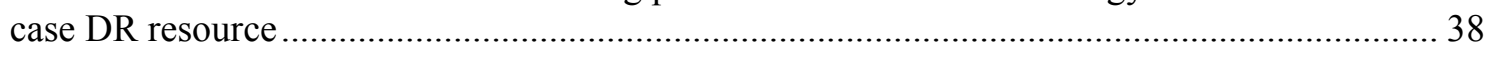

Figure 30. Breakdown of annual DR revenue for each scenario of renewables penetration

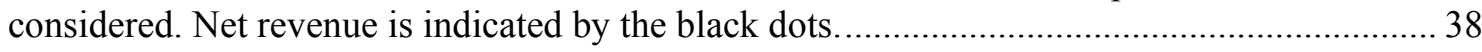

\section{List of Tables}

Table 1 Validation of GAMS Model. 


\section{Introduction}

The structures of future power systems are uncertain. Several common objectives have emerged, including the accommodation of high penetrations of renewables, increased economic and resource efficiency, and the maintenance of current reliability standards; yet, there is no consensus on how best to achieve these objectives. Across most future scenarios being explored in large-scale integration studies, the need for operational flexibility emerges as a common theme [1], [2]. Flexibility can be realized on both the supply and demand side, and even within transmission networks. The focus of this work is the modeling of energy-shifting demand response in production cost models.

Demand response (DR) is a broad term that encompasses all manners in which end-use electrical load can be altered to support the operation of the power system. It covers a range of time-scales and services, from frequency regulation, to load shifting on an hourly scale, and further on to long-term capacity provision and end-use efficiency improvements. At all of these scales, there is a general lack of accurate models depicting how DR will participate in the bulk power system. Thus, while DR appears to be a promising candidate for providing power system flexibility, its true value is as yet unknown.

Recent work has shown how DR might be incorporated into large-scale integration studies, and thus competed against other energy resources on both a capacity and an operational basis [3], [4]. The framework developed by Hummon et al. is based on DR resource data estimated using a topdown approach that applies time-varying fractional estimates of sheddability, controllability, and acceptability, to aggregate power consumption data broken down by end-use [5]. The work described in this report complements that earlier work by developing a methodological framework for bottom-up analysis of load-shifting DR from thermal-electric loads (e.g., air conditioning, water heating, heat pumps and refrigeration). For these loads in particular, it is difficult to model the physical characteristics and constraints of the underlying end-use accurately from a top-down perspective. We thus seek to more accurately model resource availability and constraints through the use of dynamic models of individual loads while respecting the mixed-integer programming (MIP) complexity of representation allowable in a conventional unit commitment algorithm. The methodology is demonstrated on the motivating example of supermarket refrigeration, which, from the perspective of DR, has the positive characteristics of high thermal mass, and potentially low enablement costs (e.g. based on largescale rollout across an entire supermarket chain).

A wealth of research addresses DR, yet the body of work concerned with establishing models suitable for its practical implementation in large-scale power system studies is limited. Many authors consider implementing detailed state-space or time-series models of flexible load directly within system dispatch algorithms [6], [7]. This approach provides an assessment of the theoretical value that DR would provide if its flexibility were fully accessible, but the complexity of this representation of DR renders it impractical. System operators do not have the ability to manage many thousands of devices, each on the scale of a few kilowatts $(\mathrm{kW})$. The value of this modeling approach for an integration study is also limited as the complexity involved precludes the assessment of the resource over the temporal and geographical scales of interest. Another commonly adopted approach is to represent the price responsivity of load through an elasticity value [8], [9]. The simplicity of this approach is appealing; however, it has been demonstrated 
that the elasticity value of electrical demand is highly variable and exhibits no correlation with the price of electricity [10]. This holds particularly for thermal-electric loads that exhibit storage properties and non-linear losses, such as those loads used for load shifting. Furthermore, there are concerns that if the price responsivity of loads can be activated, operating large quantities of price-responsive DR could introduce instability into the power system [11], [12].

Of the works that directly discuss DR models suitable for an integration study, Zerrahn and Schill [13] present the most promising approach. In [13], load shifting is modeled similarly to a battery, with a limitation on the time span over which load shifting can occur. The work presented here expands upon that work by offering a more flexible definition of the services provided to the power system by the DR resource, and by elaborating on how the seasonal characteristics of the resource affect its flexibility. Other works that investigate models of DR suitable for integration studies include [14], which focuses on the provision of frequency regulation from residential loads. Hao et al. [14] support the objective of this work by highlighting the importance of developing a compact aggregate representation of the flexibility of loads that characterizes the set of behaviors achievable while respecting the constraints of the constituent loads. Marzooghi et al. [15] model DR in combination with solar PV and storage at the transmission level. They employ a generic model of DR that has a similar structure to a battery model, but they do not elaborate on how the parameters used can be related to the physical characteristics or constraints of the underlying end use.

The work described here deviates from previous research by developing a modeling methodology for load-shifting DR that incorporates the physical characteristics and constraints of the individual end-uses within the aggregate population flexibility model. The methodology considers the seasonal variations in the resource, which have had limited treatment in previous works, an exception being the work of Hummon et al. [4] where the seasonal variation in the DR resource is incorporated directly in the top-down resource description. The developed population model is of comparable complexity to that described in [13], [15], but it offers a greater scope of flexibility, allowing multiple DR products to be offered from a given flexible load population. The model is demonstrated through a case study in which DR is implemented on a test power system that resembles that of Colorado and Wyoming, using PLEXOS, a commercial production cost modeling tool. The case study simulations span a year and facilitate analysis of the seasonal variations in the DR resource.

This work focuses solely on load-shifting DR. It is acknowledged that flexible loads are capable of providing a range of power system services including reserves and contingency, and the decision to focus on load-shifting DR was due to the complexity of this form of DR and the ability to derive a representation of other services from a load-shifting model. The representation of load shifting in a power system model is more complex than that of, for example, contingency due to the necessity of balancing any response from the flexible load with an energy recovery. The coupling of response and recovery ensures that the local operating conditions return to their normal operating level following the DR event. The load-shifting DR model fully characterizes the flexibility of the resource and consequently also facilitates the characterization of the resource available for the provision of contingency and other ancillary service support. Another reason for the focus on load shifting is that its participation in the power system can be easily understood within the framework of existing day-ahead, balancing, or real-time markets, where the system operator is free to dispatch the DR resource within its declared constraints. In 
comparison, the requirements for DR providing contingency services are not well defined. This is particularly the case for thermal-electric loads where a recovery is necessary following a response. There are no clear guidelines for how this recovery should be treated if thermal-electric loads participate in contingency support, and it is possible that these flexible loads would be precluded from contingency services due to the necessity of energy recovery.

The DR model developed in this work represents the resource as seen at the interface between the aggregator/retailer and the system/market operator. No consideration is given to how the resulting dispatch will be distributed among the constituent flexible loads in the DR population. A significant body of work discusses the scheduling of individual flexible loads; for examples see [16], [17]. Given that the physical constraints of the individual loads are reflected in the population model, it is assumed that any dispatch within those constraints can be achieved by a separate control framework at the interface between the aggregator and the device using established methods. 


\section{Preliminaries}

\subsection{Conventions and Terminology}

In this report, any references to the power adjustments that occur during a DR event are taken from the perspective of the power system. When power is supplied to the power system, this is a positive quantity that corresponds to a reduction of load, while power drawn from the power system is a negative quantity representing an increase in load.

The DR resource modeled here is considered to be comprised of two quantities: the base load and the flexible load. The base load is the consumption required to maintain normal operating conditions and is included in the conventional system load. The flexible component of the load is the adjustment away from this baseline. The flexible load is offered to the system operator as a product to be dispatched alongside conventional power system resources.

A DR event consists of a response followed by a recovery. This terminology does not have any implications for the direction of the response or recovery. A DR event can commence with either a supply of power to the system or a draw of power from the power system. At the device level, this translates to a DR event commencing with either a shed of load or pre-cooling/pre-heating.

\subsection{Running Example: Supermarket Refrigeration}

The research described here builds on previous work by the authors wherein a time series model of a supermarket refrigeration system was identified using experimental data from a refrigeration test center [18], [19]. This time series model facilitated the simulation of the refrigeration system behavior during DR events, and thus informs the DR population model described in this work. The example figures shown in Section 3 are built using the time series model that was developed in our previous work, and refer to the behavior of flexible refrigeration. The characteristics exhibited in these figures can be found across all types of flexible thermal-electric loads, though with different levels of flexibility and coupling with the environment. 


\section{Characterizing Thermal-Electric Load-Shifting Demand Response for Power System Studies}

Many thermal-electrical loads share key characteristics that make them ideally suited to providing load-shifting DR. The flexibility to operate within an acceptable temperature range and the dynamic interaction between electrical input and heat output mean that power consumption can be shifted in time while maintaining acceptable operating conditions. Such thermal loads include heating, cooling, and refrigeration, and can be found in residential, commercial, and industrial settings.

The analogy of a battery is often employed to describe load-shifting DR in power systems due to the energy storage that occurs during load shifting [20], [21]. However, there are several distinctions between batteries and appliances capable of load shifting.

\subsection{Characterizing Demand Response through the Saturation Curve}

The primary distinction between a battery and load shifting is that while a battery contains a fixed energy volume, the amount of energy stored or dissipated through load shifting is nonlinearly dependent on the magnitude of the adjustment in power consumption. The ability of flexible loads to adjust their power consumption is limited by constraints at the device level that ensure the controlled temperatures do not deviate from an accepted range; these are often called comfort or operational limits. Thus, the response provided by a flexible load is said to saturate once a temperature constraint becomes binding and the adjustment in power consumption can no longer be maintained.

The phenomenon of response saturation is illustrated in Figure 1 and Figure 2, which illustrate the behavior of a refrigeration unit when it is required to follow a power consumption reference. Figure 1 shows that the power consumption is steady until it is required to reduce from $14 \mathrm{~kW}$ to $8 \mathrm{~kW}$. The reduction of $6 \mathrm{~kW}$ can be maintained until the temperature in the refrigeration system reaches its upper bound (as seen in Figure 2). Once the upper temperature limit is reached, the prescribed reduction can no longer be maintained, at this point it is said that the response has saturated. When the power reference is no longer active, the system will recover the energy lost during the response event by increasing consumption to the maximum allowable level such that all the compressors on the system are operating to bring the case temperature back to the normal operating conditions, that is, following a temperature set-point as shown in Figure 2. This recovery is seen in Figure 1 when the power reference returns to zero. Note that in this case, the consumption at $14 \mathrm{~kW}$ is the baseline consumption, and the deviation of $6 \mathrm{~kW}$ is the response provided to the power system. 


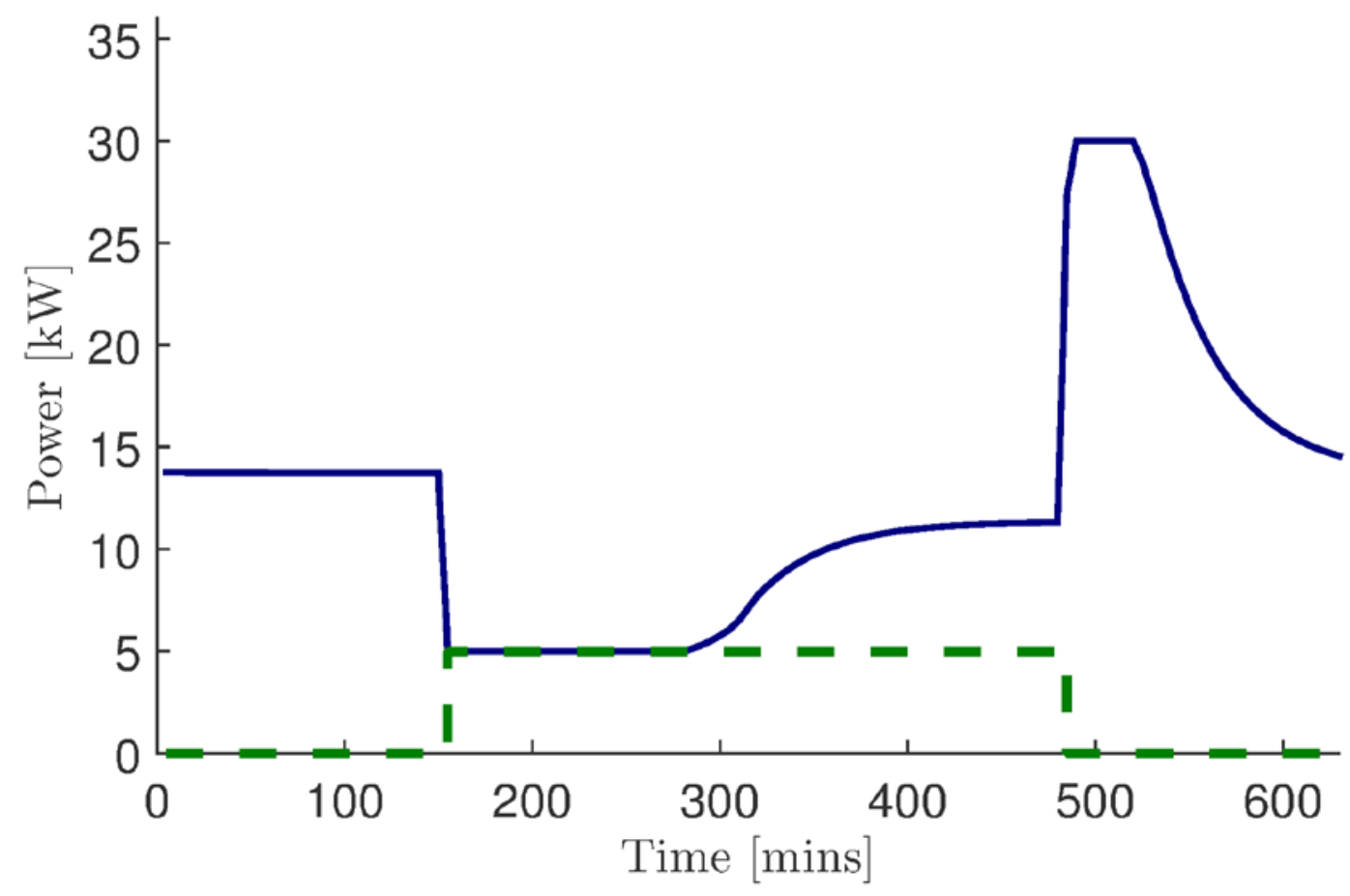

Figure 1. Power consumption in refrigeration system when required to follow a power reference (dashed green line, active when non-zero)

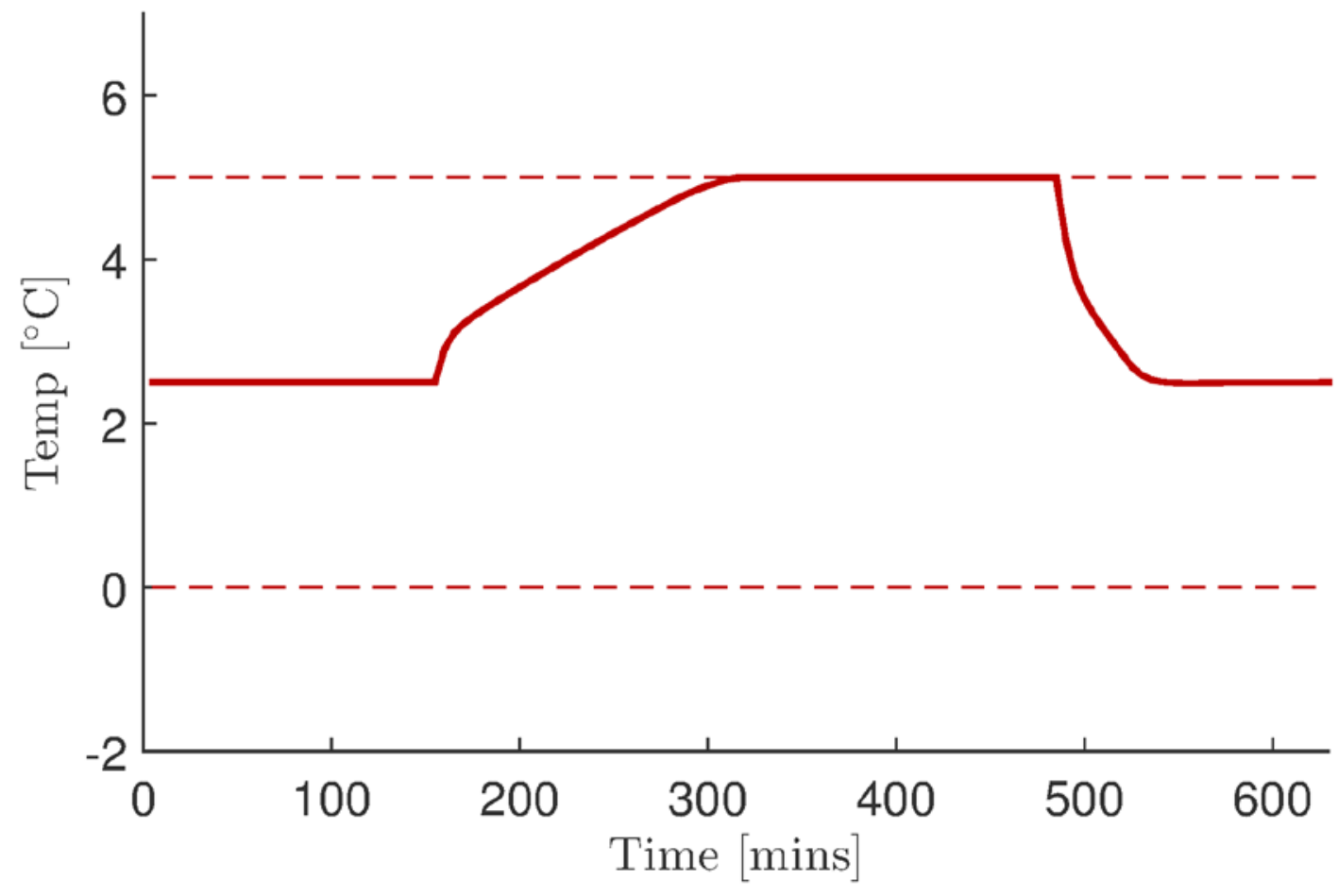

Figure 2. Temperature in the refrigeration system, subject to upper and lower limits (dashed red lines) 
Figure 3 describes the maximum flexibility of a load-shifting device in the form of a saturation curve, which illustrates the relationship between a power adjustment in a flexible load and the duration for which the adjustment can be maintained. This curve applies to a particular flexible resource or population of resources at a particular time or under a particular set of environmental conditions. Any adjustment in consumption on one side of the saturation curve (a response) must be combined with an adjustment in the opposite direction as found on the other side of $\mathrm{x}$-axis (a recovery) to complete the DR event. This ensures that the energy deviation caused by a response is corrected through a recovery. The combination of a response and recovery, possibly separated by some period of time, form a DR event. The energy stored or dissipated during this response can be calculated as the factor of the magnitude of the power adjustment and the duration for which it is maintained.

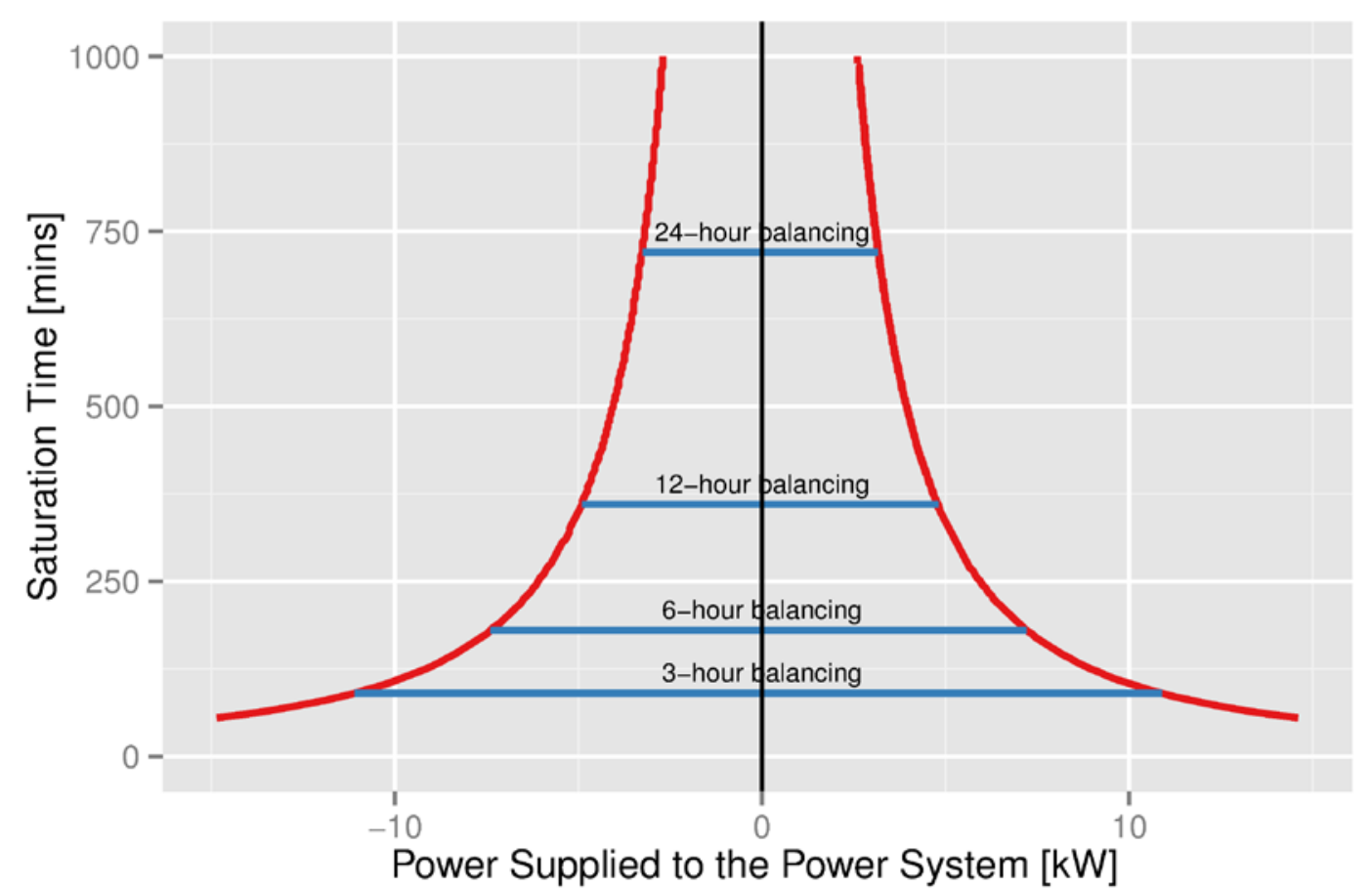

Figure 3. Saturation curve of a sample refrigeration system as seen from the power system perspective

The saturation curve provides valuable insight into the capabilities of a single load-shifting appliance, and it can similarly be employed to describe the capabilities of a population of similar appliances. For a homogeneous population of flexible loads, an aggregate saturation curve can be found through simple summation along the power axis. It is intuitive that a group of $\mathrm{N}$ identical appliances can maintain an adjustment in power consumption of $\mathrm{N \alpha} \mathrm{kW}$ for the same duration as a single appliance can maintain an adjustment of $\alpha \mathrm{kW}$. For a heterogeneous population, one can cluster appliances into approximately homogeneous sub-groups and determine an aggregate saturation curve for each. Clustering has been previously employed to represent heterogeneous populations of DR resources in [6], [14], [22]. 
While the saturation curve is an effective representation of the abilities of a flexible load, or population thereof, it is not suitable for direct inclusion in a power system model or marketclearing algorithm. The saturation curve represents a large number of combinations of upwards and downwards power adjustments, each for distinct maximum durations. While there is a relationship between each power adjustment and its saturation time, it is a non-linear relationship that cannot be linearized for inclusion in a linear optimization, such as market clearing or unit commitment.

To simplify the saturation curve sufficiently such that the characterization of the DR resource is suitable for inclusion in a unit commitment model, it is necessary to define a subset of abilities within the saturation curve. Figure 3 illustrates four possible combinations of capacity and duration that a population of load-shifting DR resources could offer into an energy market. The combinations are defined by the period within which the response and recovery must balance. Once that is specified, the maximum upwards and maximum downwards power adjustments can be read off of the saturation curve. Those, along with the balancing period, that is the sum of the saturation times for the maximum upwards and downwards power adjustments, are used to define the DR thermal storage resource within the unit commitment model. Thus, the saturation time (y-axis value) shown for each selection in Figure 3 is 50\% of the DR storage resource's balancing time. Imposing a balancing period ensures that the underlying flexible load returns to an intermediate temperature following a DR event, so that it is prepared for future events. The DR storage resources are mutually exclusive; only one choice of balancing period can be actively dispatched at a time.

\subsection{Resource Efficiency}

A further distinction between load-shifting DR and a battery is that the round-trip efficiency of a DR event is dependent on the magnitude of the response and recovery. In a conventional battery or energy storage device the efficiency is considered constant and independent of the charging/discharging rate.

The efficiency of a load-shifting DR event can be calculated as the ratio of the energy supplied to the power system and the energy drawn from the power system during a DR event.

$$
\eta_{D R}=\frac{\sum_{t=1}^{T} \Delta P^{+}}{\sum_{t=1}^{T} \Delta P^{-}}
$$

Determining the round trip efficiency of a DR event is non-trivial, as the efficiency depends on the magnitude of both the response and the recovery. Based on the models developed in [18], the efficiency profile of DR events from a supermarket refrigeration system has been calculated and is shown in Figure 4. Figure 4 illustrates the efficiency of DR events that commence with power drawn from the power system (above) and power supplied to the power system (below). The efficiency has been calculated by combining response and recovery events sampled from the saturation curve. The energy associated with each response and recovery has been calculated from the power magnitude and duration of each.

A higher efficiency $(>100 \%)$ is most desirable, as this indicates that the amount of energy drawn from the power system is less than the energy supplied to the system. It can be observed from Figure 4 that the efficiency can range between approximately $60 \%$ and $180 \%$, depending on the 
magnitude of the response and recovery. Events in which the power supplied to the power system are small but sustained for a long time, and the power drawn is large but over a short time, exhibit the highest efficiencies.

This can be understood by considering our running example of a refrigeration system. A power draw from the power system to the refrigeration system reduces the temperatures within the refrigeration system and consequently increases the losses to the system. A prolonged power draw will result in a slow reduction in temperature and large energy losses, requiring more power be drawn from the grid and consequently reducing the efficiency of the DR event. In contrast, a large power draw will reduce the temperature rapidly and can only be maintained for a short period due to saturation. This behavior results in reduced losses to the ambient and improved DR efficiency.

Symmetric events (where response and recovery have the same magnitudes) tend to exhibit an efficiency of just below $100 \%$. The efficiency of symmetric events is indicated in Figure 4 by the yellow line, while the $100 \%$ efficiency contour is indicated in red. It can be observed that while the efficiency of a symmetric event is close to $100 \%$, it does not exhibit a fixed offset from the $100 \%$ efficiency contour and is dependent on the magnitude of the response and recovery.

We did not consider the possible variation in the efficiency with changing environmental conditions because requisite data were not available. This is a possible topic for future research. 

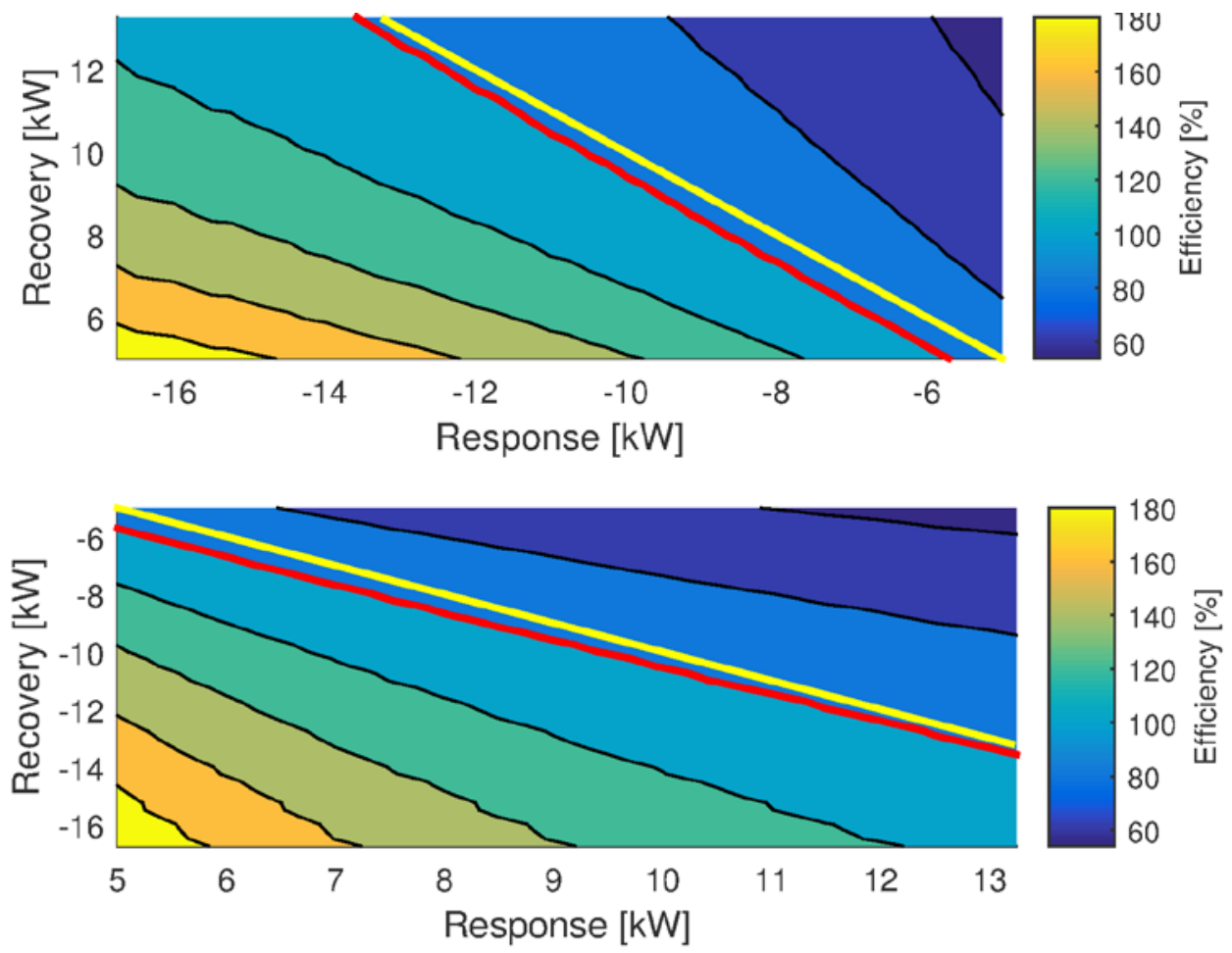

Figure 4. Round-trip efficiency of a DR event

The red lines show the $100 \%$ efficiency contours and the yellow lines show the efficiency of symmetric events (i.e., a response and recovery of the same power magnitude).

\subsection{Seasonality in the Demand Response Resource}

When considering thermal end-end uses for the provision of load-shifting DR, there are three key characteristics to consider; the baseline power consumption, the maximum possible power consumption, and the energy required to achieve a given temperature change. These quantities are influenced by several environmental factors - primarily the ambient temperature - but also occupancy and typical building operating hours, among other factors.

The baseline power consumption defines the amount of power consumption that can be shed as part of a DR event. Figure 5 illustrates the normalized power consumption of a number of thermal end uses over a year. The data are sourced from the California Commercial End-use Survey (CEUS) [23], which recorded hourly power consumption of commercial loads in California throughout 2002. The dependence of the baseline consumption on the ambient temperature can be clearly seen in Figure 5. For heating and cooling, this variation in consumption is attributable to both the effect of ambient temperature on the performance of the appliance, and the reduced use of cooling devices in the winter and heating devices in the summer. For refrigeration and hot water, the usage is steady year-round, but the impact of the ambient temperature on appliance performance can be seen. It is tempting to conclude that the couplings of refrigeration and hot water heating, and of cooling and heating are well suited to act together to provide a DR resource with stable availability over the year; however, the significant differences in magnitude reduce their complementarity. 


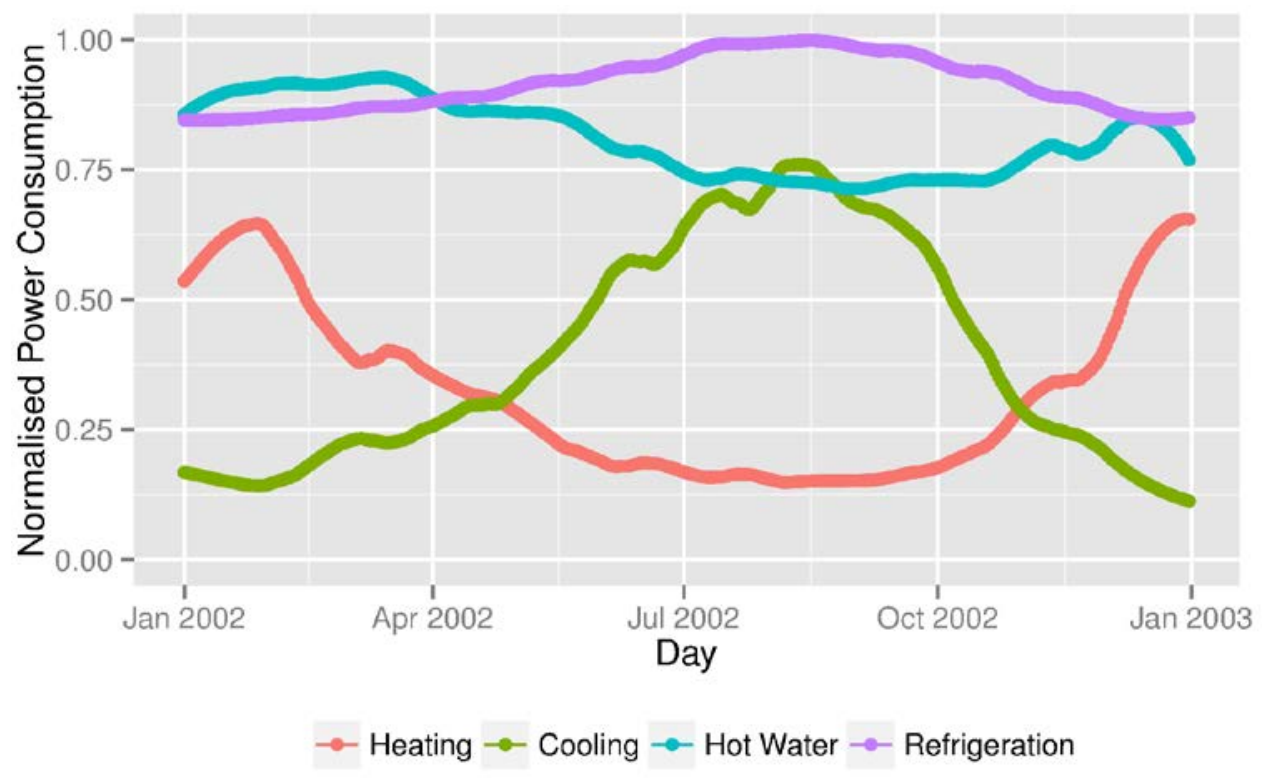

Figure 5. Normalized consumption of electrical end uses suitable for load shifting

Data have been smoothed and normalized to facilitate the analysis of the seasonal tendencies of each end use without considering their individual magnitudes. Intra-day variations in the power consumption profiles cannot be seen in the illustrated data, as they have been averaged out in the smoothing process. Normalization is relative to the maximum daily power consumption, the proximity of the illustrated profiles to 1 indicates the level of intra-day variations in the data.

The difference between the baseline power consumption and the maximum possible power consumption defines an upper limit on the power that can be drawn from the power system during a DR event. Figure 6 illustrates the baseline and maximum power consumption for the refrigeration system used in the running example in this work. Due to the characteristics of the compressors on the refrigeration system, this maximum power consumption increases during the summer. Details on the derivation of the maximum and baseline consumption are provided in Section 5.1. It can be seen that the baseline consumption peaks during the summer, indicating an increase in capacity available to supply power to the power system. However, the difference between the baseline and maximum consumption is minimized during the summer. This means there is a reduced ability to recover energy during a DR event, which possibly limits the value this DR resource offers the system during the summer months. 


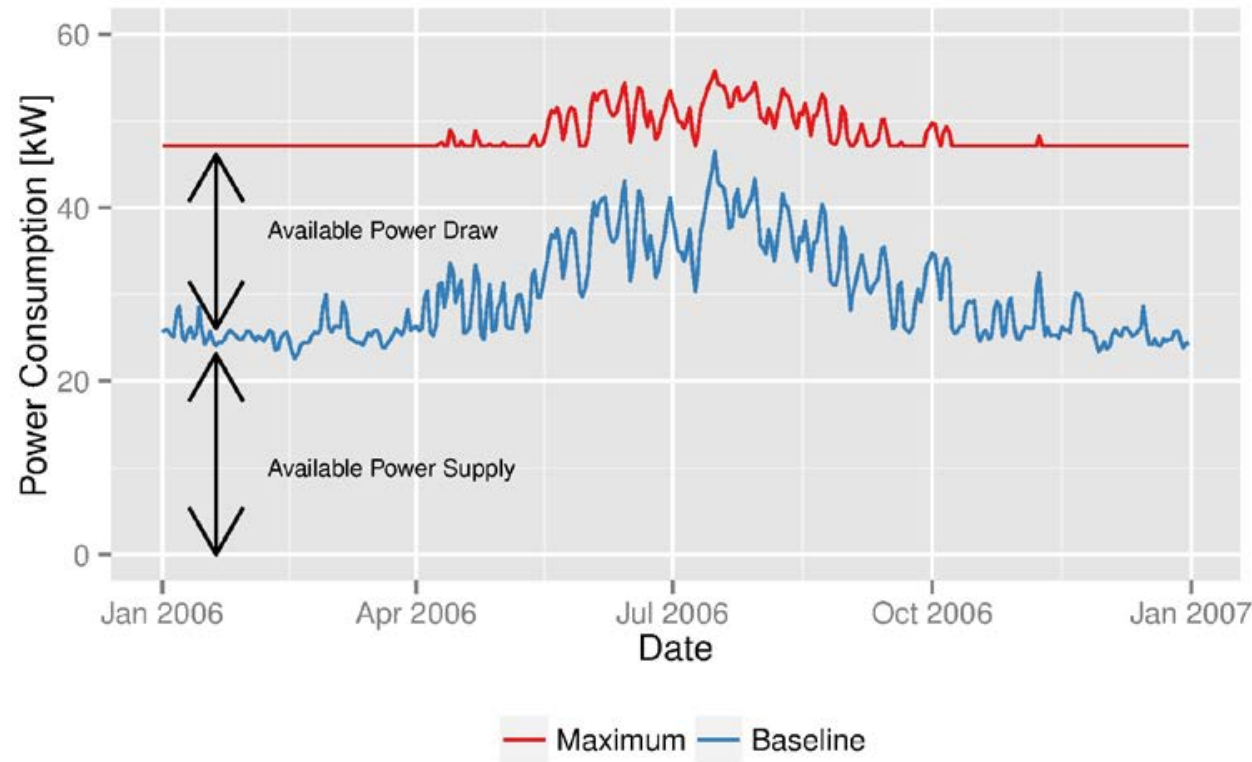

Figure 6. Variations in available flexibility at the device level, using the example of supermarket refrigeration

The energy required to achieve a given change in temperature also varies over the year for a number of thermal end-uses. The coefficient of performance (COP) is a temperature-dependent quantity that defines the relationship between power consumption in a thermal appliance and the resulting thermal energy. At high COP values, a smaller amount of power consumption will be necessary to achieve a given temperature change. This affects the definition of a DR product by varying the maximum response and recovery power for a product with a fixed balancing time. Figure 7 illustrates the COP of the refrigeration system employed as the running example in this work. The COP of this system is maximized during the winter months, indicating less response and recovery power will be offered in the DR products during the winter.

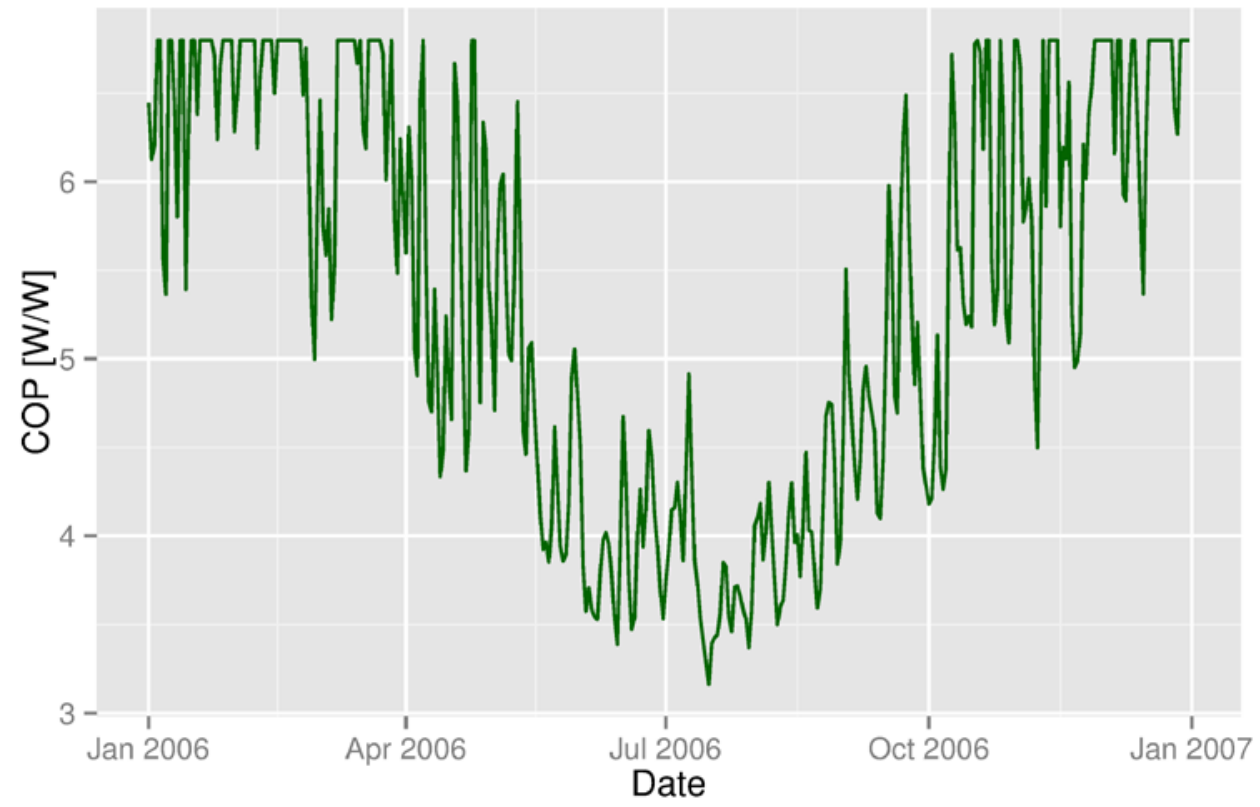

Figure 7. Variations in compressor COP over a year 


\section{Mathematical Representation of Demand Response Resource}

Though Section 3 described the differences between load-shifting DR and a battery, their similarities are sufficient to use a battery or conventional electrical storage formulation to represent the load shifting resource in a power system model. This section details the theoretically optimal mathematical formulation for this resource, as well as a simplified representation suitable for inclusion in commercial production cost modeling software. The mathematical formulation describes the constraints on a DR resource offered by a population of flexible loads. Each resource offered consists of a maximum amount of power supplied to or drawn from the power system, and a period over which the power supply and draw must balance. Examples of products are illustrated in Section 3.1.

\subsection{Theoretically Optimal Formulation}

The load-shifting DR event consists of a combination of power supplied to and drawn from the power system. At the device level, this is seen as a deviation in power consumption from a timevarying baseline. Equation (1) describes the power supplied to the power system, $\Delta P_{t}^{+}$, and the power drawn from the power system, $\Delta P_{t}^{-}$, as the difference between the baseline power consumption of the population of flexible loads, $P_{t}^{B A S E}$, and its actual consumption, $P_{t}^{D R}$. Both $\Delta P_{t}^{+}$and $\Delta P_{t}^{-}$are positive variables; thus, when the actual consumption of the population of flexible loads is less than its baseline, power is supplied to the power system and $\Delta P_{t}^{+}$is nonzero. Similarly, when the actual consumption exceeds the baseline, power is drawn from the power system and $\Delta P_{t}^{-}$is non-zero. The baseline power consumption is not considered part of the load shifting resource; it is assumed to be served as part of the conventional load.

$$
\begin{gathered}
\Delta P_{t}^{+}-\Delta P_{t}^{-}=P_{t}^{B A S E}-P_{t}^{D R} \\
0 \leq \Delta P_{t}^{-} \leq \Delta P_{t}^{-, \max } u_{t} \\
0 \leq \Delta P_{t}^{+} \leq \Delta P_{t}^{+, \max } u_{t}
\end{gathered}
$$

Equations (2) and (3) include the binary variable $u_{t}$, which is employed to indicate whether a DR product is active. The necessity of this variable is clarified below. 
The storage characteristics of the load shifting resource are described below.

$$
\begin{gathered}
S_{\mathrm{t}}=S_{t-1}+\left(\Delta P_{t}^{-}-\frac{\Delta P_{t}^{+}}{\eta^{D R}}\right) \cdot \Delta t \\
S_{\mathrm{t}} \leq S^{\text {Balance }}+\Delta S^{+, \text {max }} \\
\mathrm{S}_{\mathrm{t}} \geq S^{\text {Balance }}-\Delta S^{-, \text {max }} \\
\Delta S^{+, \text {max }}=\sum_{t \in \text { Balance }} \Delta P^{-, \text {max }} \cdot \Delta t, \quad \Delta S^{-, \text {max }}=\sum_{t \in \text { Balance }} \Delta P^{+, \text {max }} \cdot \Delta t \\
S_{t}-S^{\text {Balance }} \leq M u_{t} \\
S_{t}-S^{\text {Balance }} \geq-M u_{t}
\end{gathered}
$$

Equation (4) describes the stored energy, $S_{t}$, as a function of the inflow to and outflow from the storage device, where $\Delta t$ is the interval. The stored energy has no direct physical relationship with the underlying flexible load. The storage analogy is simply employed to indicate that the energy level returns to the balance point, $S^{\text {Balance }}$, when the energy supplied and drawn from the power system balance. The value of $S^{\text {Balance }}$ is arbitrary, but must be sufficiently large that maximum energy outflow over the balance window does not exceed $S^{\text {Balance }}$. If this is not considered, the lower bound on the storage volume (i.e. zero) may become binding. The lower and upper bounds on the storage device are given in equations (5), (6), and (8), which describe the maximum possible energy deviations from the balance level over the balance window for a given DR resource configuration.

The parameters constraining the behavior of the DR resource, $\Delta P^{+, \max }, \Delta P^{-, \text {max }}$ and $P_{t}^{\text {Base }}$ are time-varying to reflect the change in available DR resource and the variations in the underlying flexible load over the year.

Equations (8) and (9) are coupled constraints used to indicate when the storage level is not at the prescribed balance point. When the stored energy deviates from the balance point, the binary variable, $u_{t}$, becomes non-zero. $\mathrm{M}$ is an arbitrarily large number that ensures the constraints are non-binding when $u_{t}$ is non-zero.

The load-shifting product is considered to be online when either power is supplied or drawn from the power system, or when the stored energy is different from the prescribed balance level. When a product is online $u_{t}=1$.

$$
\sum_{t^{\prime}=t}^{t+T^{\text {Balance }}} u_{t^{\prime}} \leq T^{\text {Balance }}, \forall t
$$


The constraint that the DR product must balance within the prescribed balance window is imposed in equation (10), which states that a given product may be online for at most the balance period, $T^{\text {Balance }}$. This ensures the product goes offline once the balance period is exceeded. Requiring that the product be offline consequently imposes that the power supplied to and drawn from the grid during the active period of the product are balanced. This constraint is comparable to a maximum uptime constraint for a conventional generator.

The binary variable employed to indicate the online status of a DR configuration, $u_{t}$, can also be used to ensure a given DR resource is not simultaneously offering two configurations to the power system. Each DR configuration allows the maximum use of the resource flexibility, so simultaneous dispatch of multiple configurations would result in the dispatch of services that are not achievable without violating the temperature constraints of the underlying thermal load. If each DR configuration is denoted using the subscript $i$, each variable in the above equations should be extended to incorporate this additional subscript. The set of DR products is denoted $I$.

The constraint preventing simultaneous dispatch of DR configuration is given as:

$$
\sum_{i \in I} u_{t, i} \leq 1, \quad \forall t
$$

The use of the binary variable also lends itself to the definition of additional constraints that could be imposed if desired by the aggregator, retailer, or other party responsible for control of the flexible load. One such constraint is an upper limit on the number of DR product activations within a given window:

$$
\begin{gathered}
S U_{t}-S D_{t}=u_{t}-u_{t-1} \\
\sum_{t \in T \text { period }} S U_{t} \leq X
\end{gathered}
$$

Equation (12) defines the startup and shutdown indicators for a given DR product. Equation (13) then limits the number of startup instances over a given period, $T^{\text {period }}$, to less than a $\operatorname{limit} X$.

Constraining the number of DR events dispatched within a given period is a useful construct to ensure flexible loads are not inadvertently driven toward unacceptable operating conditions. The concept of linking the response and recovery is intended to ensure the operating conditions (e.g., temperature) return to their initial states following a DR event. This assumption holds if the constant efficiency of the demand response event assumed in the optimization problem formulation is accurate. However, as described previously, the efficiency is dependent on the magnitude of the response and recovery. Including this dependency in a linear programming optimization is not possible, thus a constant value must be selected. By selecting demand response products in which the response and recovery are of equal magnitude, it can be assumed that $100 \%$ efficiency is a reasonable approximation (see Figure 4). However, the DR dispatch algorithm has the freedom to select response and recovery magnitudes below the prescribed maximum levels, which may not be $100 \%$ efficient. In such a case, the operating temperatures would not return to their initial levels at the end of the DR event. Initiating a second DR event 
from this initial point would possibly lead to unforeseen saturation. Limiting the number of events dispatched would allow more time for the flexible load to return to its normal operating conditions outside of the DR event.

The constraints described above have been formulated in an efficient manner for mixed-integer programming using insight acquired from [24].

\subsection{Practically Implementable Formulation Suitable for PLEXOS}

The constraints described in the Section 4.1 represent the ideal control of the DR products; however, they are not directly implementable in the selected production cost modeling software, PLEXOS. In particular, equation (10) requires the summation of variables over time, which cannot be implemented in PLEXOS.

Equation (10) ensures the DR resource will balance within the prescribed balance period following its initialization at an arbitrary time. This can be simplified by instead requiring the DR resources to balance at or prior to a set time. For example, a resource with a three-hour balancing period can be required to balance at 3 a.m., 6 a.m., 9 a.m., and so on.

The variable $u_{t, i}$ is a custom binary variable that is defined in PLEXOS. Each DR product ( $i$ ) within each population of flexible loads has an associated binary variable $u_{t, i}$ for each time period $(t)$. The constraints detailed in the previous section ensure $u_{t, i}$ is non-zero any time a DR product is online, that is, when it is supplying power to the system, drawing power from the system, or has a stored energy value not equal to the balanced energy value.

\subsection{Validation of Approach}

Implementing the DR resource within PLEXOS requires the constraint simplification described above; however, prior to doing so, it is important to establish the impact of this simplification on the problem solution.

Validation of the constraint simplification has been achieved by comparing the results of a PLEXOS model with simplified DR constraints, and an equivalent model implemented in GAMS with the ideal DR constraints. The General Algebraic Modeling System (GAMS) is a high-level modeling system for mathematical programming problems [25]. A simple five-bus system model in PLEXOS was replicated in GAMS. The models were validated with and without DR as described by the simplified constraints. Finally, the ideal DR constraints were implemented in GAMS.

The production cost model dispatches the sample five-bus power system on a day-ahead basis over a year. The system contains five thermal generators and no variable renewable generation. Both models use a duality gap of $0.01 \%$. PLEXOS uses the XpressMP solver to determine the model solution, while GAMS uses the CPLEX solver.

Table 1 details the total production cost from the GAMS and PLEXOS implementations with and without DR, and the GAMS implementation with the ideal DR constraints. Differences between the GAMS and PLEXOS solutions are expected due to the different solvers used. A difference of $0.02 \%$ in the total production cost is seen between the GAMS and PLEXOS solutions, both with 
simplified DR constraints and with thermal generation only. This difference is considered to be within the tolerance for validation. The addition of the ideal DR constraints in the GAMS formulation reduces the cost of production by a further $0.014 \%$ compared to the simplified constraints as implemented in GAMS.

Table 1. Validation of GAMS Model

\begin{tabular}{lc}
\hline Model & Total Production Cost (\$M) \\
\hline PLEXOS & 158.282 \\
GAMS & 158.318 \\
PLEXOS with Simplified DR & 157.834 \\
GAMS with Simplified DR & 157.799 \\
GAMS with Ideal DR & 157.775 \\
\hline
\end{tabular}

The value of DR in this test case is the reduction in total production cost achieved by its implementation in the system. The PLEXOS model reports a cost reduction of $0.282 \%$, while the GAMS model reports savings of $0.33 \%$ with the simplified DR representation. Again, the differences can be attributed to differences in the solvers used to generate the solution. The implementation of the ideal DR constraints in GAMS results in total cost savings of $0.34 \%$. Implementation of the ideal DR constraints results in additional savings of $4.52 \%$ compared to the simplified DR constraints. While these additional savings are not insignificant, they are sufficiently small that the use of the simplified constraints in PLEXOS can be justified.

In addition to being implementable in PLEXOS, the simplified constraints significantly reduce computation time. A yearlong simulation in GAMS with simplified constraints on a five-bus system has a run-time of 0:07:59, while a simulation with the ideal constraints has a run-time of 0:32:06. ${ }^{1}$ Given that these simulations are conducted using a very simple power system model, one would expect the significant difference in computation time to be greatly increased in a larger, more complex system.

\footnotetext{
${ }^{1}$ Simulations were run on a Dell laptop running Windows 7 with an Intel(R) Core i7-3520M CPU at 2.9Ghz and 4GB of RAM.
} 


\subsection{Example Operation in a Five-Bus System}

Figure 8 illustrates the results of a small test case run on the five-bus system using PLEXOS. Three DR populations of varying sizes were modeled with four possible balancing periods each, 3-hour, 6-hour, 12-hour, and 24-hour (defined as illustrated in Figure 3). The system was simulated over a one-week period at hourly resolution to illustrate the operation of the load shifting DR resource. Because the choice of balancing on six-hour intervals was not dispatched in the simulated period it is not shown in Figure 8.

The upper plot in Figure 8 shows the electricity price over the simulation period. The higher prices approximately correspond to the periods of peak load during each day. The second plot shows the net load reduction of each DR resource, as defined by the balancing period. By comparing the first and second plots, it can be seen that the DR resource provides net power to the system during periods of higher prices and recovers energy during periods of lower prices.

The differences between the various balancing periods offered to the system are also evident in the second plot of Figure 8, where the 24-hour balancing period is associated with the lowest power magnitude but is active for a much longer duration than any other resource configuration. The dashed lines represent the power limits on each of the demand response resource configurations. The variations of these limits with ambient temperature can be seen; for example on January 3, the limits are greater than on the other days due to higher ambient temperatures.

When the DR event commences with a supply to the grid (e.g., the first DR event in Figure 8), at the appliance level the event starts with a shed of load and is followed by a rebound or recovery. When the event commences with a draw of power from the grid, the appliance first undertakes a pre-heating or pre-cooling cycle that pre-charges the thermal storage resource, and only later sheds load to provide energy to the power system. In most cases, the DR event consists of a continuous response followed by a continuous recovery; however, in the first instance of use of the 12-hour balancing period configuration, the DR event consists of a repeated oscillation between supply and draw of power. This is a valid DR behavior that is permitted within the defined characteristics of the DR resource as presented to the grid model.

The impact of the simplified constraints imposed in PLEXOS can also be seen in Figure 8. For example, the 24-hour balancing period configuration must balance prior to midnight, but it is also possible for the 12-hour balancing period configuration to be active between the time when the 24-hour resource balances and its own balancing deadline occurs, which is also midnight in this case. This can be seen in the repeated pattern from January 4 to January 7.

The third plot in Figure 8 illustrates the energy volumes involved in each DR event. Here the difference between the DR configurations is again evident, with the longer balancing period exhibiting larger volumes of energy storage. The final plot indicates the online status of each of the configurations offered, illustrating that while the DR resource is presented to the grid model in a multifaceted manner, no two conflicting configurations are ever dispatched simultaneously. 

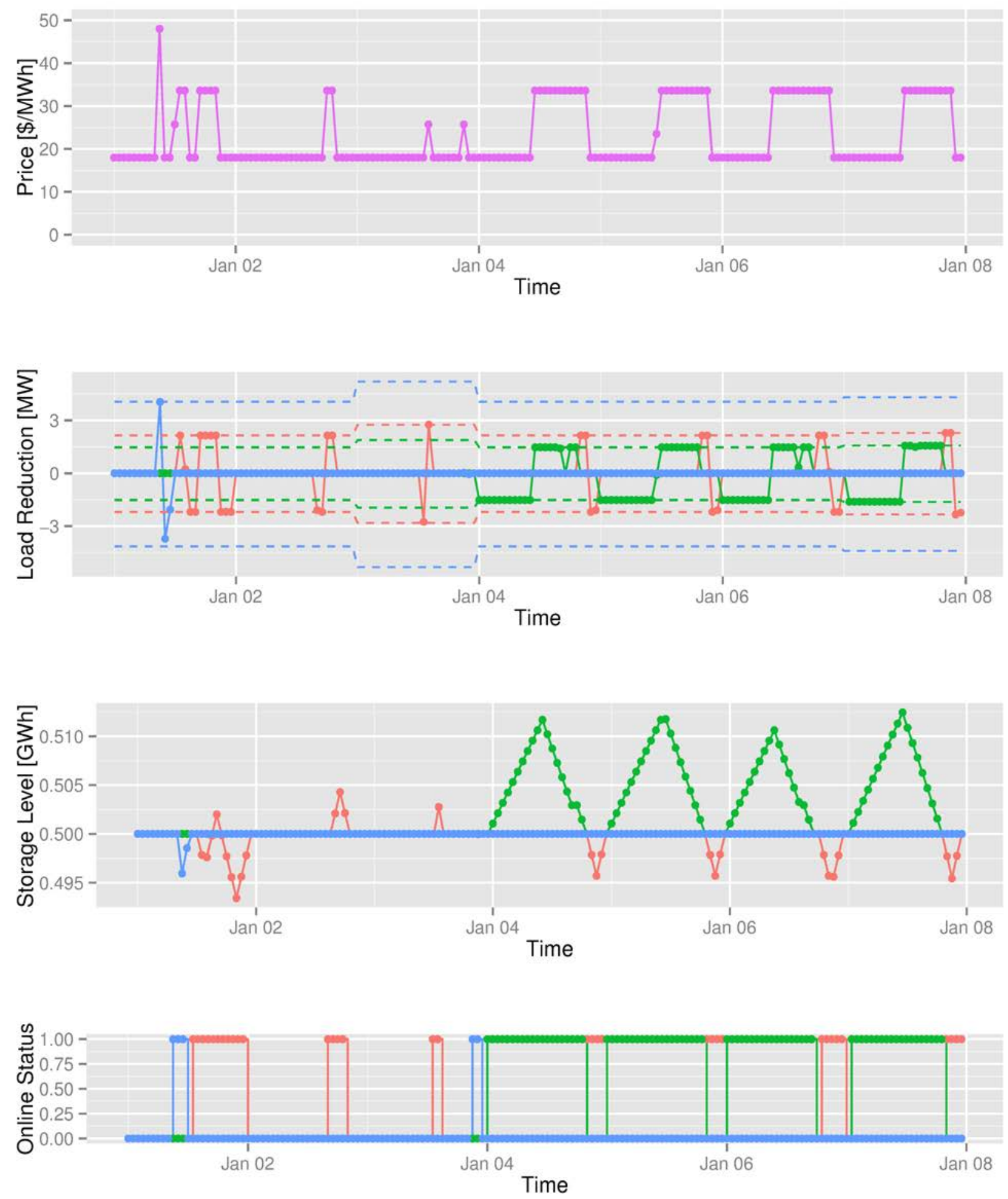

DR Configuration $\rightarrow$ 12hr Balance $\rightarrow 24 \mathrm{hr}$ Balance $\rightarrow 3 \mathrm{hr}$ Balance

Figure 8. DR resource behaviour over a one-week period as simulated on a five-bus power system model 


\section{Case Study}

We conducted case studies to demonstrate the DR modeling methodology described above and to investigate the impact of DR on the power system. This section details the development of a sample population of DR resources, the power system within which the DR resource is implemented, and the tests that are performed.

\subsection{Demand Response Resource}

The sample DR resource implemented in these case studies is supermarket refrigeration. DR is achieved by altering the compressor operation, which is precipitated by adjusting temperature set-points within refrigerated cases. This resource did not include defrost, display case lighting or anti-sweat, other potential sources of DR that can be leveraged.

Supermarket refrigeration exhibits several characteristics that make it ideally suited to being an early adopter of load-shifting DR:

- The thermal mass present in refrigeration display cases facilitates the adjustment of power consumption while maintaining acceptable temperatures for foodstuff storage.

- Supermarkets operate at a low profit margin, incentivizing them to pursue opportunities for cost savings. If DR can offer easily accessible cost savings, it can be expected that this profit-driven enterprise would adopt an operating paradigm that facilitates loadshifting DR. This differs from residential DR, where consumers are not rational actors and may be driven by many other factors than welfare maximization.

- The structure of a supermarket chain lends itself to the formation of an aggregator. While individual supermarkets are considered large commercial loads, the flexibility they offer is likely below the threshold for participation on many electricity markets. By aggregating a number of supermarkets and offering their combined flexibility as a single product, this threshold can be overcome.

To represent the load shifting flexibility of supermarket refrigeration accurately, it is necessary to establish the saturation characteristics and power consumption limitations of the individual supermarkets. A saturation curve for a sample supermarket has been established in previous work [18], [19]. A portfolio of tests was conducted at an experimental refrigeration facility, mimicking the behavior of a supermarket during a DR event. The data resulting from these tests were used to identify a statistical model of the system that was in turn employed to establish the saturation curve. In practice, the same saturation curve can be determined directly through observing the ability of a supermarket to follow a power reference signal.

The model employed to establish the saturation curve does not consider the dependence of the system on the outdoor ambient temperature due to the limited time extent of the available experimental data. This necessitates the consideration of the temperature dependence through an external model.

Three key temperature-dependent quantities have been determined: the baseline power consumption, the maximum power consumption, and the COP. These quantities have been 
identified using an operational supermarket located in Denver, Colorado as a base case. The precise location of this supermarket cannot be revealed due to commercial sensitivity.

The baseline consumption has been obtained from historical data recorded at the supermarket. A regression model relating the power consumption to ambient temperature was identified and used to simulate the baseline consumption for the case study year, 2006.

The maximum power consumption is not a measured quantity. The maximum power consumption to achieve the required refrigeration cooling is detailed in the data sheets for the compressors on the refrigeration system. The specifications of the compressors were employed to simulate the maximum power consumption over the study year.

The COP of the refrigeration system is a well-defined quantity. Its relationship to the ambient temperature is detailed in the compressor specifications, and can be described by a non-linear regression model. This model facilitated the simulation of the COP over the study year. The variations in the COP impact the DR product definition, as the maximum power offered for response and recovery vary according to the COP.

The COP can be incorporated into the saturation curve by considering that a fixed amount of thermal energy is required to achieve a given change in temperature, and that the electrical energy required to achieve this temperature change will change according to the variation in the COP. The saturation curve identified for the test refrigeration system was found from data recorded at an ambient temperature of $0^{\circ} \mathrm{C}$, a temperature at which the COP is at its maximum value. The saturation curve at any other temperature can then be found by scaling the base saturation curve along the power axis according to the change in COP. Figure 9 illustrates the difference between the saturation curve at maximum and minimum COP values for the study year.

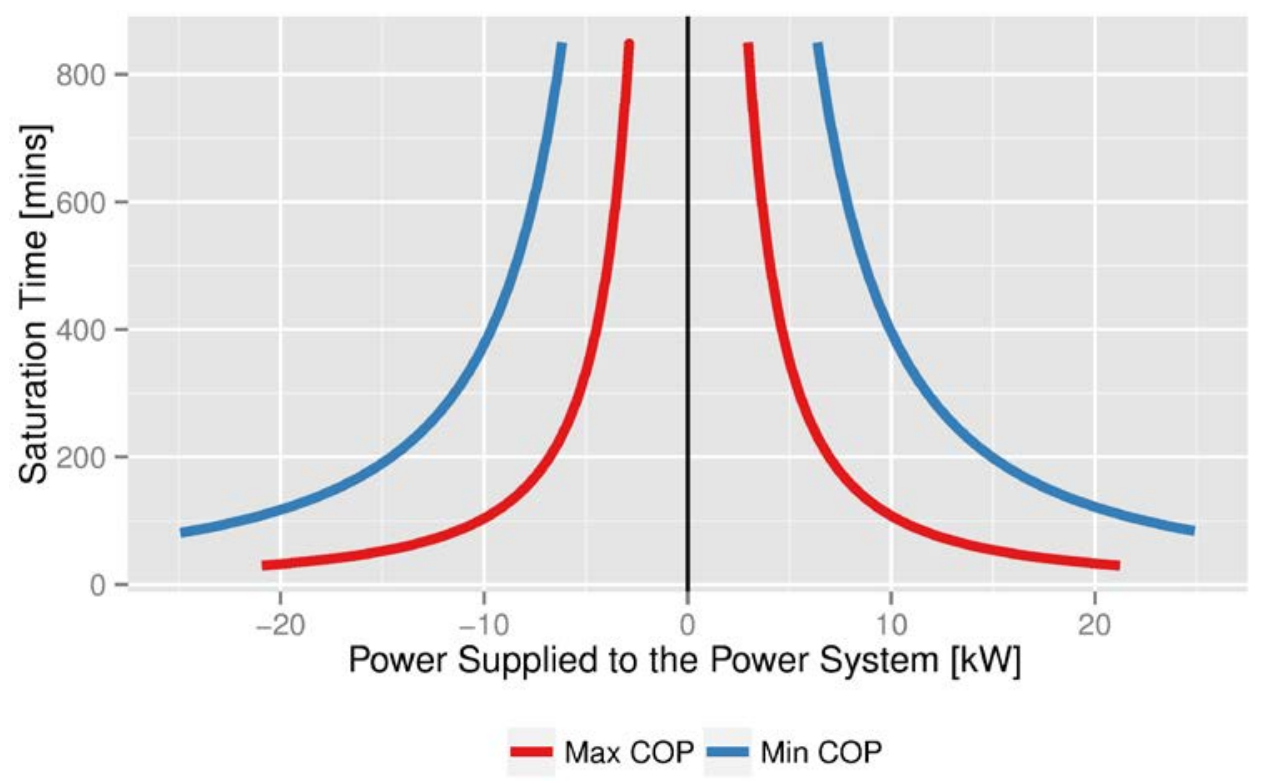

Figure 9. Impact of COP variations on the saturation curve 
Variations in ambient temperature are considered at a daily resolution, as considering hourly changes in temperature would require a significant modeling effort to isolate the effect of ambient temperature on the baseline power consumption from other factors, including store opening hours. Ambient temperature is the only external factor considered for the model, as there is a lack of available data on other factors that influence the power consumption. Further work could be conducted to acquire data and model the impact of other factors, including opening and closing hours, and stocking schedules.

\subsection{Population Building}

The models described above provide a representation of the flexibility of a single, sample supermarket. This base model has been used to construct a population of supermarkets representing the population of all supermarkets in Colorado.

The population of supermarkets is divided into three sub-populations: small, medium, and large stores. The supermarkets are assumed to be homogeneous within each sub-population. This facilitates the calculation of an aggregate saturation curve representing the dynamics of the each sub-population. For each sub-population, the saturation curve and power flexibility limits of the base model have been scaled appropriately. The average baseline consumption of small, midsized, and large supermarkets is assumed to be $30 \mathrm{~kW}, 50 \mathrm{~kW}$, and $80 \mathrm{~kW}$, respectively. ${ }^{2}$ The baseline and maximum consumption of the small supermarket has been illustrated in Figure 6 (above).

The saturation curve is adjusted for each population by shifting the curves along the power axis. This adjustment assumes that larger or smaller supermarkets will contain the same type of display cases (which contain the thermal mass providing the storage/flexibility) but will have more or fewer of them. Thus, for a given balancing period (e.g., three hours), the power offered by the large supermarket sub-population will be greater than that of the mid-size or small supermarket sub-population as there are more compressors on the system that will adjust their power consumption and achieve the same temperature change in each of the associated display cases.

The structure of each of the sub-populations was informed by a combination of data from the Commercial Building Energy Consumption Survey (CBECS) [26] and the County Business Patterns (CBP) data set [27]. CBECS provides detailed energy micro-data on a small population of commercial businesses across the United States. The CBECS data set only considers a small set of supermarkets, with locations indicated at the resolution of census regions. A census region is typically a group of states. Using the CBECS data, it was possible to determine a link between the floor size of a supermarket and the number of employees. The CBP data set contains less information on each supermarket, but includes all supermarkets and information on the number of employees in each, with their location indicated at the county level. Taking the number of employees as a proxy for store size, it was possible to determine the number of small, medium, and large supermarkets in each county in Colorado.

\footnotetext{
${ }^{2}$ The size of the sample supermarket within each sub-category has been informed by discussions with industry experts and analysis of power consumption data from a number of supermarkets located around the United States. The data employed to determine the power consumption characteristics of each sub-population cannot be shared directly as they are commercially sensitive.
} 
As the overall flexibility resource in each population is quite small, it was decided to consider a single population encompassing all supermarkets in Colorado, divided into the aforementioned sub-populations. The population is comprised of 482 small supermarkets, 178 mid-size supermarkets and 140 large supermarkets.

\subsection{Test System Description}

To analyze the impact of DR on power systems, it is necessary to employ a model that is large enough to be realistic, but small enough to facilitate repeated simulations and sensitivity studies with reasonable run times. The test system employed in this work was developed for previous integration studies conducted at the National Renewable Energy Laboratory (NREL) [4], [28], [29]. It is based on a subset of the U.S. Western Interconnection consisting of two balancing areas located in and around Colorado: the Public Service Company of Colorado (PSC) and the Western Area Colorado Missouri (WACM). This test system was derived from a database constructed by the Western Electricity Coordinating Council (WECC) Transmission Expansion Policy Planning Committee (TEPPC) and from other publicly available data sets. The system is modeled zonally; PSC and WACM are individually modeled as copper plates, with transmission linking the two regions. The system is simulated using projected values for load and renewable resources in the year 2020. The system is a summer-peaking system with a peak load of 13.7 GW, and annual demand of $79 \mathrm{TWh}$. The region modeled is primarily comprised of vertically integrated utilities, whose interactions and behaviors are complex and difficult to model. For simplicity, it is assumed that the system as a whole is dispatched for a least-cost solution. Further information on the test system employed in these studies can be found in [29].

The DR resource represents a very small proportion of the test system, though determining its precise capacity is complicated. The amount of power supply available to the power system can be easily calculated; however, the need to balance the energy supplied to the system within a given time often limits the amount of power that can feasibly be supplied to the system. Figure 10 plots the penetration of the available load reduction from the DR resource as a percentage of the rest of the generation capacity on the system. It is clear that the population of DR resources representing all supermarkets across Colorado makes a very small contribution to the overall power system, never exceeding a capacity share of $0.25 \%$. Determining an accurate capacity value for DR is complicated, and other works have attempted to establish this metric [3].

Figure 11 illustrates the available DR flexibility. It can be observed that when the power supply is at its peak, the available power draw is at its minimum point. This supports the theory that the requirement to balance power supply and draw within the balance window may limit the power supplied to the power system. The power supply corresponds to the baseline consumption of the flexible population; this quantity can be shed, effectively supplying power to the power system. The power draw corresponds to the difference between the baseline consumption and the maximum possible consumption. This quantity is the additional power that can be consumed by the flexible load (i.e., drawn from the power system) to recover energy during a DR event. 


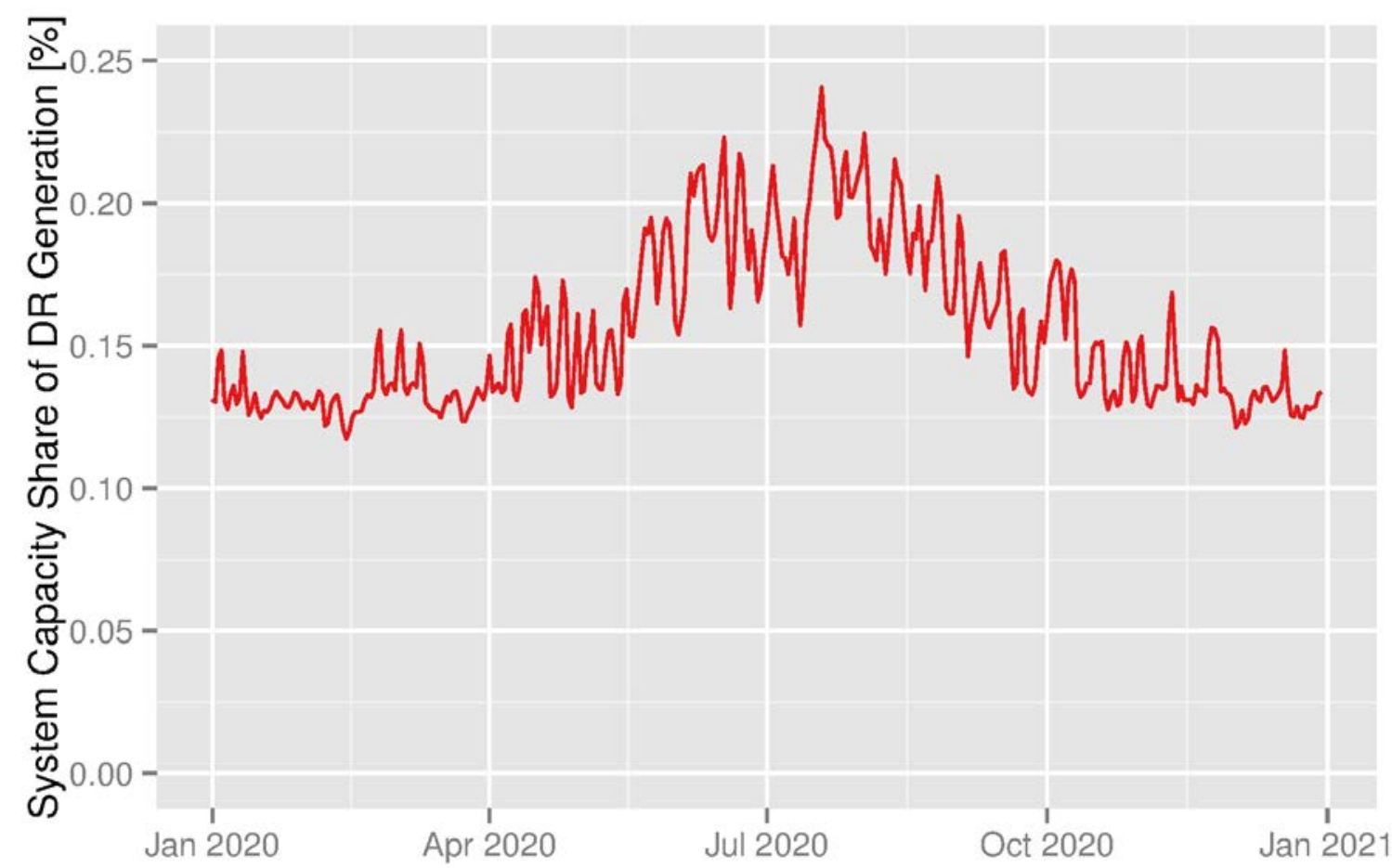

Figure 10. DR generation share of system capacity

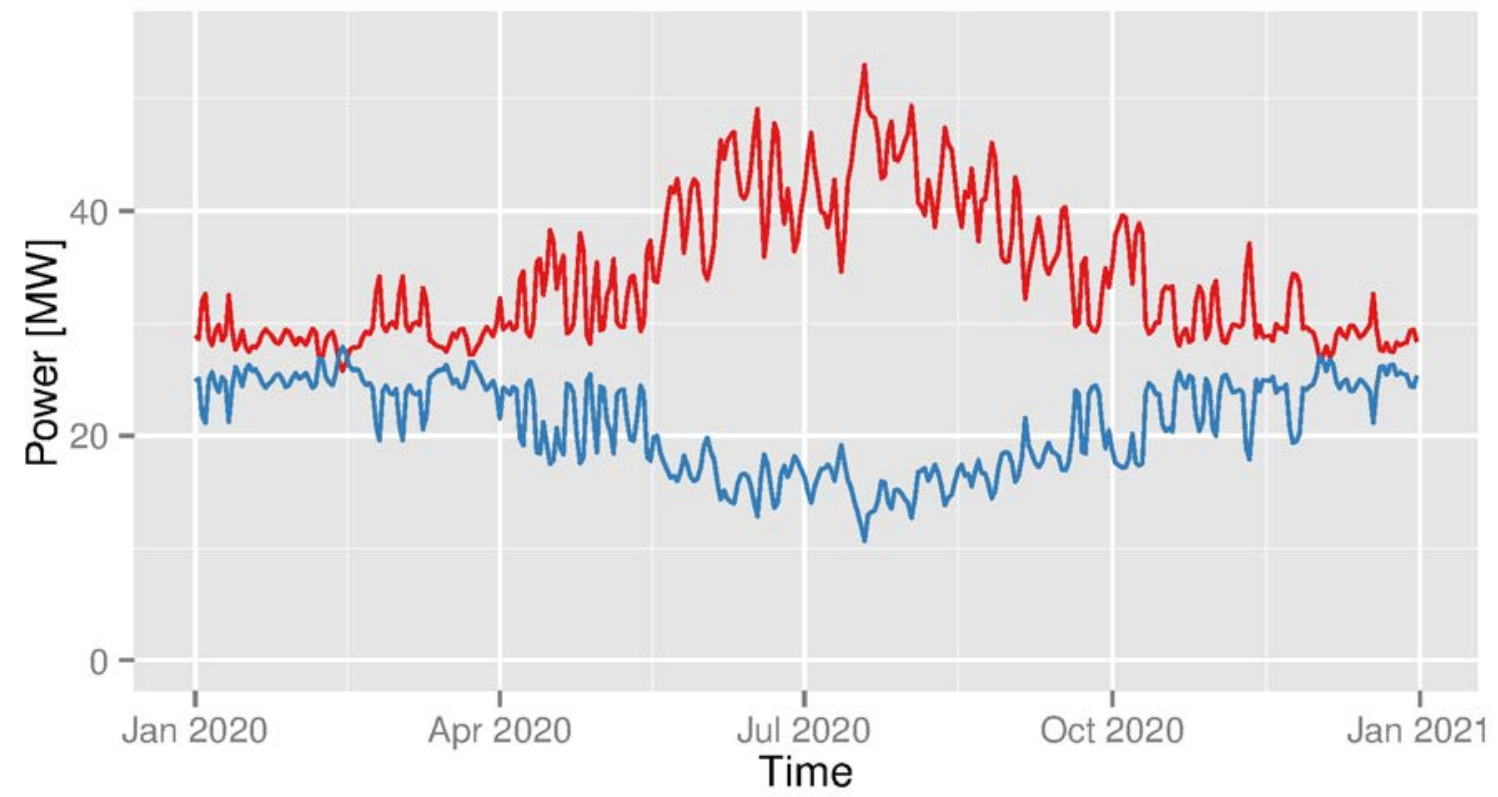

- Power Supply — Power Draw

Figure 11. Available DR flexibility in the study year (2020) 


\subsection{System Dispatch Framework}

In the case studies, we assess the value of DR for providing flexibility through real-time load shifting. Flexibility is the ability of the power system as a whole to react to forecast errors for base load and variable generation from renewable resources. The ability to react to contingency events (e.g. the failure of a generator or transmission line) is managed through the reserves. The ability of DR to provide reserves is not considered in this work.

To assess the value of DR for providing flexibility, the system is initially committed on a dayahead basis, using day-ahead forecasts of load and renewables. The day-ahead commitment determines the level of generation from each generator at an hourly resolution for the coming 24 hours, and uses a look ahead for a further 24 hours at three-hour resolution to facilitate the scheduling of storage units and inflexible generators.

Load-shifting DR can be dispatched either day-ahead or close to real-time. In this work, the decision was made to focus on DR as a real-time flexibility resource. Thus, in the models considered here, DR is only dispatched at real-time, when forecast errors for load and variable generation are revealed. Demand response is not included in the day-ahead commitment process.

The system is re-dispatched close to real-time using updated forecasts for load and variable renewable generation, and including the DR resource. The real-time dispatch occurs every hour with 15-minute resolution, with a 24-hour look ahead at 3-hour resolution. The look ahead is necessary to ensure the DR products can balance by the prescribed time. The commit status of inflexible generators, including coal and combined cycle plants is held from the day-ahead commitment. The inflexible generators are permitted to deviate from their day-ahead power dispatch, though any deviation in excess of $10 \%$ on either side of the day-ahead dispatch is penalized. This structure is intended to approximately replicate real-time competitive electricity markets, where inflexible generators can only offer a limited amount of their capacity for realtime dispatch. The inflexible generators account for approximately $50 \%$ of the generating capacity on the system.

As the objective of these case studies is to establish the value that DR offers to the power system, DR is priced as a zero-marginal cost resource. Thus, the generation it provides to the power system generates revenue at the system price, and power that is drawn from the power system is priced at the system price and must be purchased. This is not intended to reflect the true cost of DR but to facilitate the assessment of its value to the system, which can then be used as a benchmark to justify, or otherwise evaluate, the investment required to establish and operate DR as a power system resource.

\subsection{Base and Sensitivity Studies}

The case study is conducted primarily to determine the impact of DR on the operation of the power system and the value it offers. In the base case, the peak generation available from DR is $63.5 \mathrm{MW}$, and the penetration of variable renewable generation is $16 \%$ on an annual energy basis. As the DR resource represents a very small proportion of the power system, it is unlikely that any significant impact on system dispatch will be visible. As such, sensitivity studies are conducted to assess the impact of this resource as its system share grows. 
The DR resource included in the base studies represents the flexibility of all supermarkets across Colorado, but it does not include other similar resources, such as refrigerated warehouses.

Additionally, the representation of the DR resources in the form of storage configurations with different balancing periods is sufficiently generic that it could reasonably be used to represent the flexibility of a much wider range of loads offering load shifting, for example air conditioning and water heating. Thus, it is reasonable to conduct sensitivities on the size of the DR resource, though an upper bound on potential resource has not been evaluated. Sensitivities are conducted considering that the DR resource is scaled by multiples of 5,10 , and 25 .

Sensitivity studies on the penetration of variable renewable generation (wind and solar PV) are also conducted. Previous studies have highlighted the increasing value of storage with increasing penetrations of variable renewables [28]. As DR exhibits several characteristics in common with conventional energy storage, increases in the penetration of renewables are expected to similarly increase the value of DR to the power system. Following the convention used in previous studies conducted with this test system, the penetration (by energy) of wind and solar PV is increased from approximately $16 \%$ (base case penetration) to $35 \%, 45 \%$, and $55 \%$. A ratio of $5: 1$ wind to solar PV is maintained in all cases. 


\section{Results}

\subsection{Base Case}

The value of DR is defined by the amount by which it reduces the cost of serving the system load. In the case studies considered here, DR is only dispatched at real-time and therefore contributes toward the balancing of forecast errors in load and renewable power generation. The metric employed here to define the value of DR is the difference between the cost of system dispatch adjustment at real time, with and without DR. In general, the cost of real-time system dispatch adjustment can be interpreted as the cost of having uncertainty in the system forecasts. By adding free DR resources, we can determine the maximum possible amount by which DR might be able to reduce these (partially unavoidable) costs.

In the base case, DR reduces this cost of uncertainty by $4.8 \%$. The cost of uncertainty accounts for $3.1 \%$ of total system costs without DR, and $2.9 \%$ with DR. In absolute terms, the base case DR resource is found to reduce operational costs by $\$ 2.089$ million in the test year. This corresponds to $\$ 32.85 / \mathrm{kW}$-year, the value per unit of DR load reduction capacity available, or $0.014 \%$ of total system operation costs without DR. Thus, this base DR resource offers a very limited benefit to the power system, but this is expected given that the resource is very small. Section 6.2 details the change in DR value at varying resource sizes.

Figure 12 illustrates the profile of the value of DR over the study year, 2020. As expected, the seasonal availability of the DR resource is reflected in this profile. Refrigeration DR offers greater potential capacity to the power system during the summer months, but its ability to draw additional power to recharge its thermal storage resource is limited. Therefore, it is expected that the overall ability of the DR resource to support system operation will be reduced during the summer months. This can be observed in Figure 12 where the value of DR is at its minimum point during the summer months of July and August.

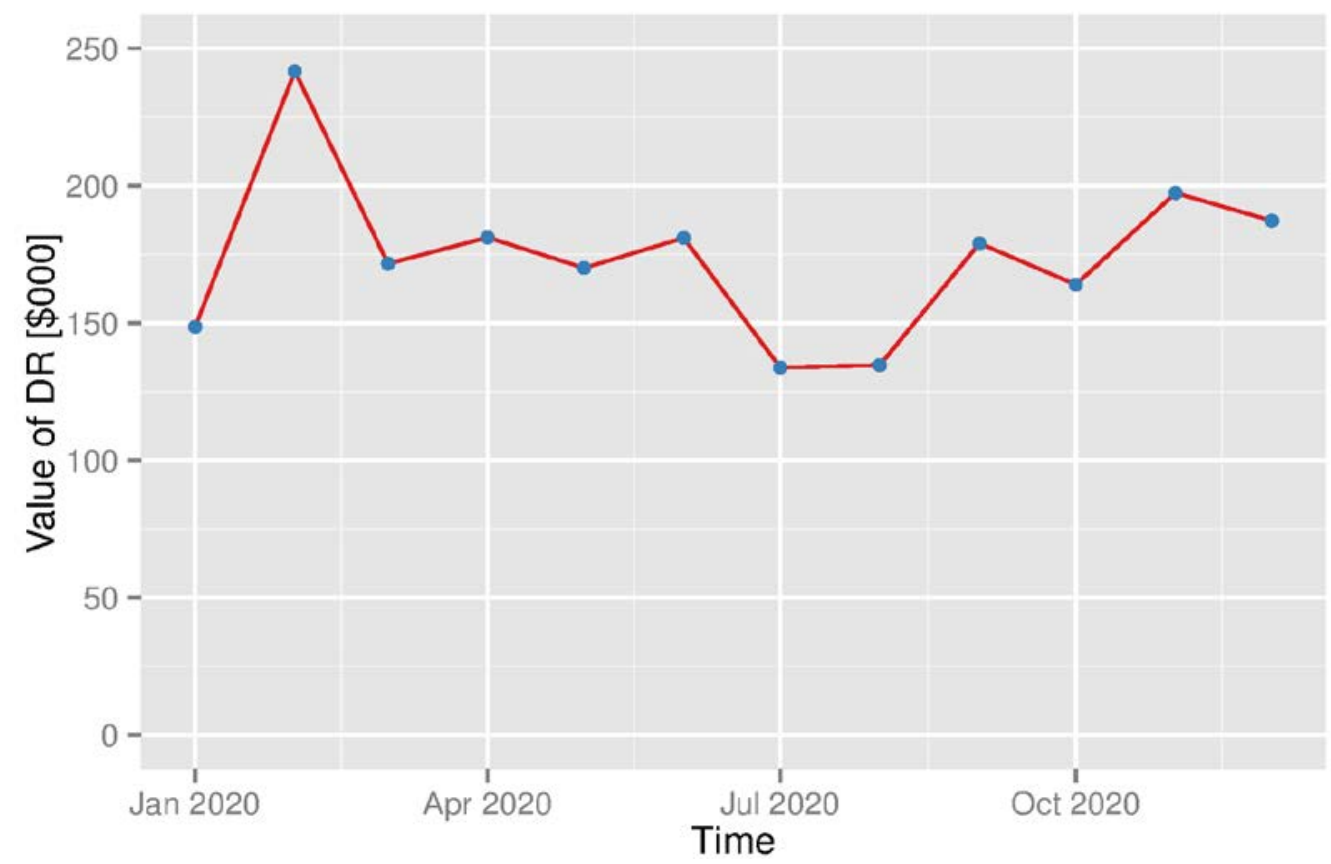

Figure 12. Monthly value of DR over the study year 
The reduction in system costs brought about through the introduction of DR can be attributed to the reduced dependence on higher cost generation and the reduction in renewable generation curtailment that occurs at real-time dispatch. Curtailment occurs due to an excess of nondispatchable renewable generation and the inability of the system to adjust the output of inflexible generators in close to real-time. Figure 13 illustrates the amount of wind curtailment that is avoided by DR per month. The total avoided curtailment over 2020 is $693 \mathrm{MWh}$, representing a reduction in curtailment of $7.2 \%$ versus the real-time dispatch without DR.

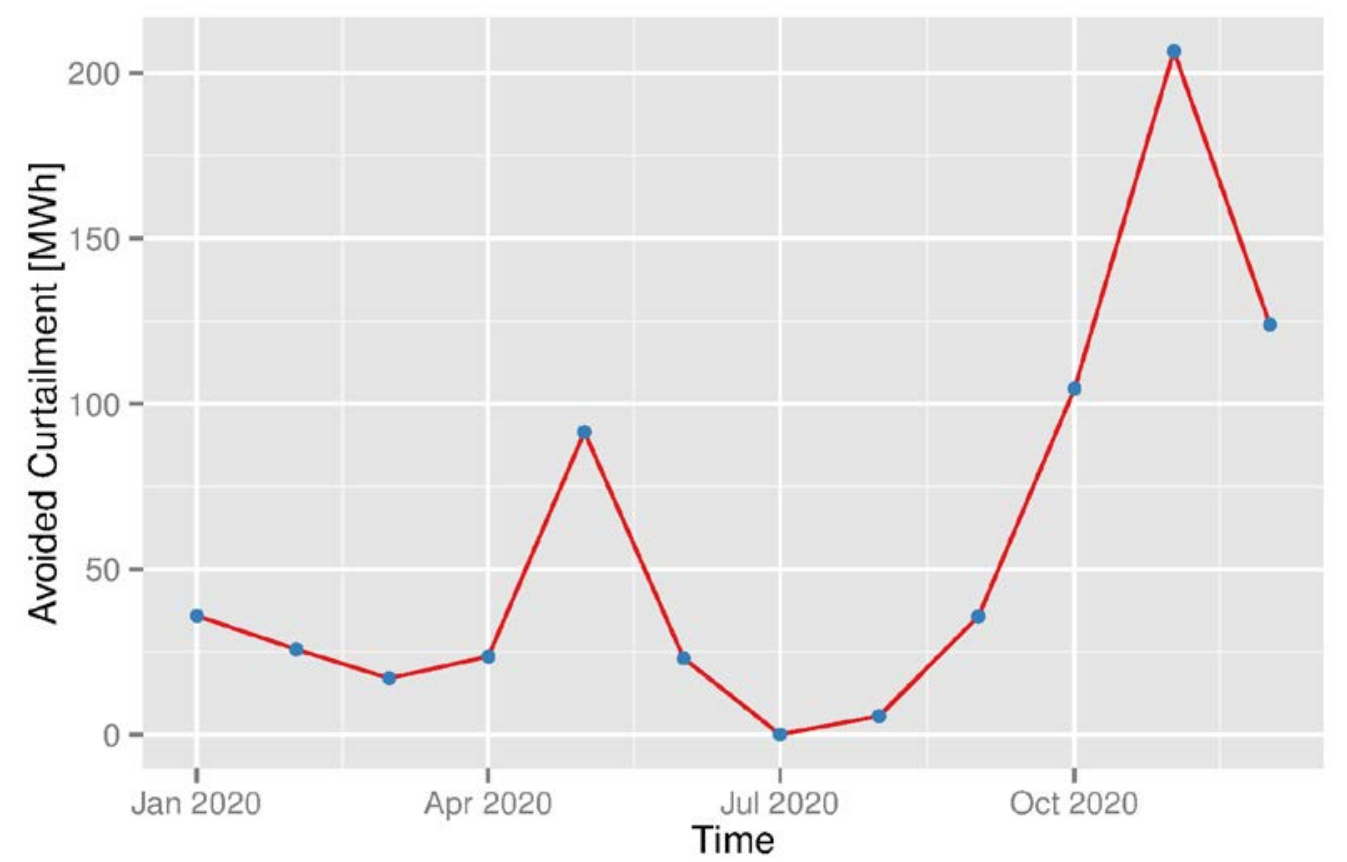

Figure 13. Monthly avoided curtailment of renewables achieved through the implementation of DR

The DR resource is comprised of three populations corresponding to different supermarket sizes. Each population offers four mutually exclusive storage configurations, demarked by balancing period length, for dispatch. Figure 14 illustrates the distribution of net revenue of the DR resource across the offered configurations. The net revenue is comprised of the income generated from the sale of power to the power system and the cost of recovering energy; both are priced at the system price. For the purpose of this case study, no operational costs are modeled for the DR resource, neither in the offering of its services into the system nor in the revenue calculation. The 24-hour balancing period configuration generates the greatest net revenue in this case. This is due to its ability to arbitrage over an entire day, taking advantage of the full range of diurnally varying system prices. It is more difficult to generate revenue over shorter balancing periods, as they rely on price differences over a smaller window. This is reflected in Figure 14 where the three-hour and six-hour balancing period configurations generate the least net revenue. The price on the test system is primarily determined by fuel costs; on other systems where the price includes other factors such as carbon costs, there may be a greater opportunity to arbitrage across greater price differences, resulting in increased revenue for DR. 


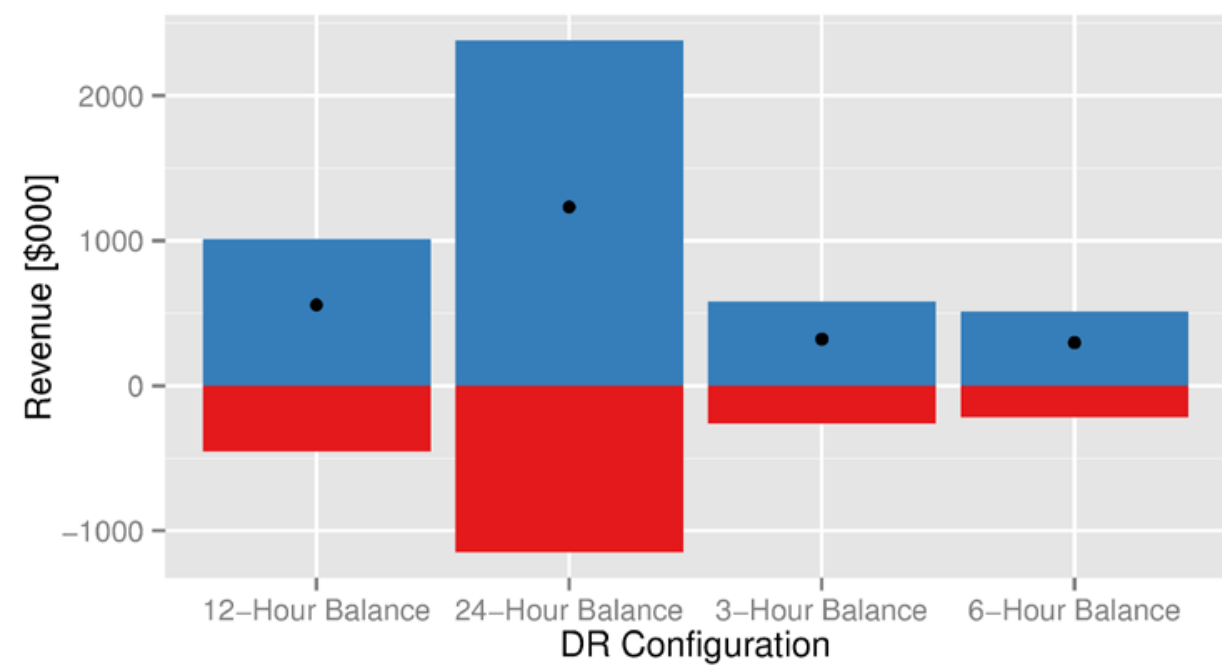

Energy Recovery Cost Energy Supply Revenue

Figure 14. Revenue breakdown by DR resource definition offered for dispatch

Net revenue is indicated by the black dots.

Figure 15 illustrates the profile of net revenue for the entire DR resource over the year.

Comparing Figure 15 to Figure 12, we can see that the profile of net revenue does not exhibit the same clearly understandable seasonal variation as the profile of the value of DR. In fact, although the value of the DR to the system is at its minimum point in July (see Figure 12), the net revenue generated in July is one of the highest, and is greater than the revenue generated in any of the winter months during which the available DR resource is greatest. Net revenue does not exhibit the same dependence on the availability of the resource as the value of DR to the power system, as it has greater dependence on price variations.

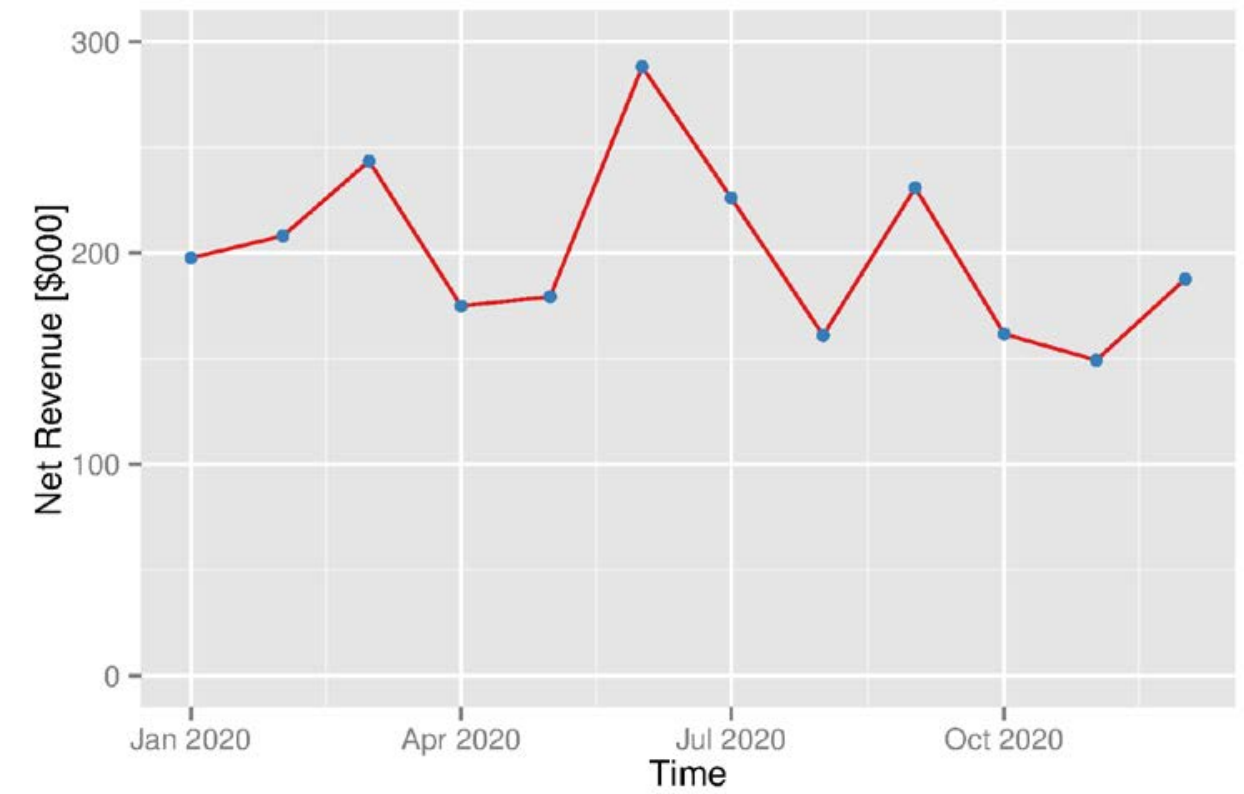

Figure 15. Monthly net revenue of the entire DR resource over the study year 


\subsection{DR Capacity Sensitivities}

In the following analyses, the impact of DR is often termed relative to the enabled DR capacity, or on a per-supermarket basis. The base DR resource is considered to have a load reduction capacity of 63.5 MW. This corresponds to the maximum theoretical amount of power by which its load could be reduced. This maximum occurs when the underlying refrigeration systems are operating at their upper power consumption limit and shed their entire load (turn off completely). Note that in this case it would not be possible for the supermarket to recover this lost energy. The sensitivity studies consider increases in the DR resource by factors of 5, 10, and 25, corresponding to enabled DR capacities of $318 \mathrm{MW}, 635 \mathrm{MW}$, and 1,587 MW. The capacities of individual small, medium, and large supermarkets are $56 \mathrm{~kW}, 93 \mathrm{~kW}$, and $149 \mathrm{~kW}$, respectively.

Figure 16 illustrates the value of DR per enabled megawatt of capacity. The marginal value can be seen to decrease as the capacity increases. This indicates that the early adopters of loadshifting DR represent a significant additional value to the system, but as more supermarkets enter the DR market, the added value of each additional supermarket is less. At the lowest penetrations of DR, a large supermarket has a value of $\$ 4,890$ per year; however, this decreases (following the trend shown in Figure 16) to only $\$ 1,030$ per supermarket at the highest investigated penetrations of DR. A small supermarket offers an annual value of approximately $\$ 500$ at that penetration level.

The manner in which the DR capacity sensitivities are conducted here is quite naïve, assuming that all of the flexible loads exhibit the same characteristics as the base DR portfolio. In reality, a larger DR resource will incorporate a diverse range of flexible loads. The diversity of a realistic DR portfolio will likely contribute to alleviating the steep decrease in the value of DR that is exhibited in our naïve sensitivity studies.

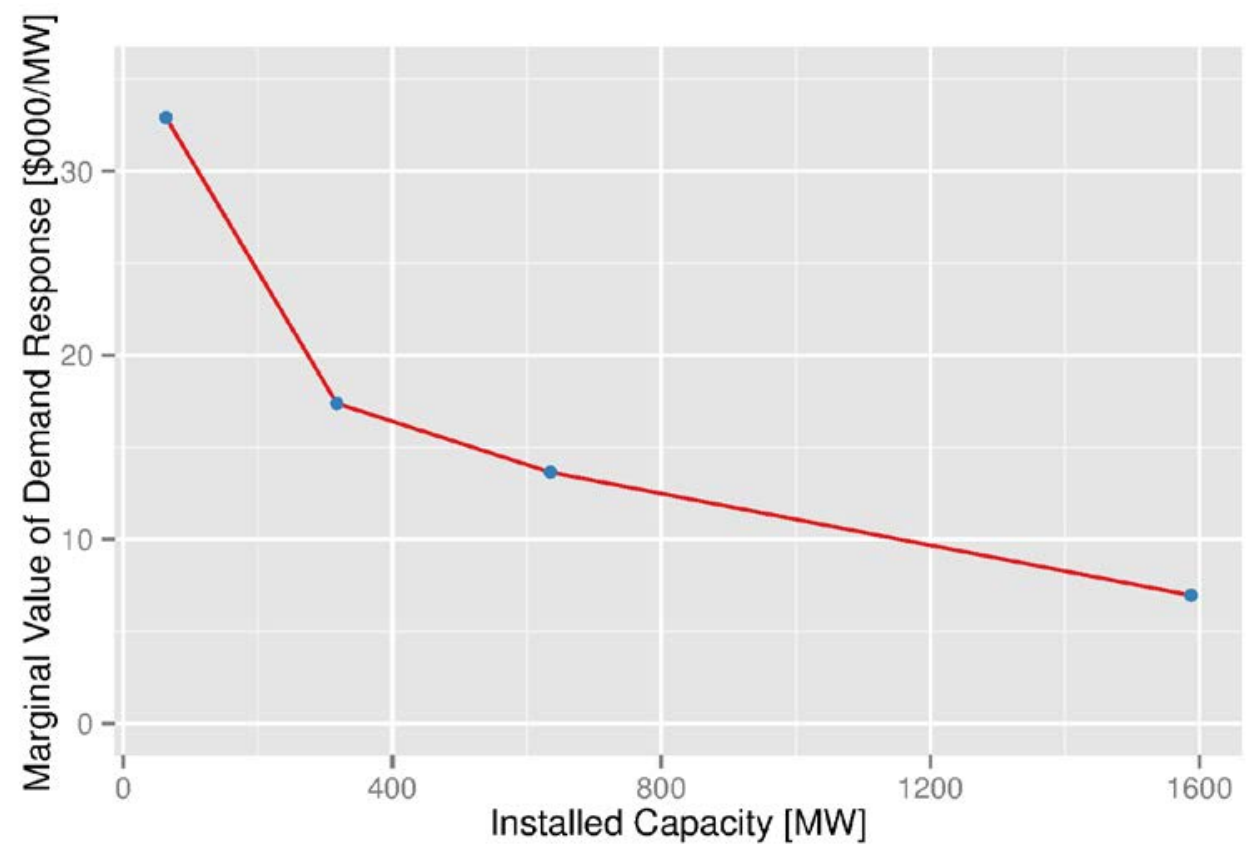

Figure 16. Annual value of DR per MW of installed DR capacity 
Figure 17 illustrates the decrease in the net revenue per enabled megawatt of DR capacity, while Figure 18 illustrates the same quantity on a per-supermarket basis. The revenue per supermarket is of a similar magnitude to the value it offers to the system. Further research is necessary to determine the capital and operational cost of this DR resource, as this must be subtracted from the net revenue presented here to determine the actual net revenue to the supermarket operator. Additionally, the system operator may offer incentives to support demand response, which can also be considered when assessing expected net revenue. It can be anticipated that any incentives will not exceed the value that DR offers to the system.

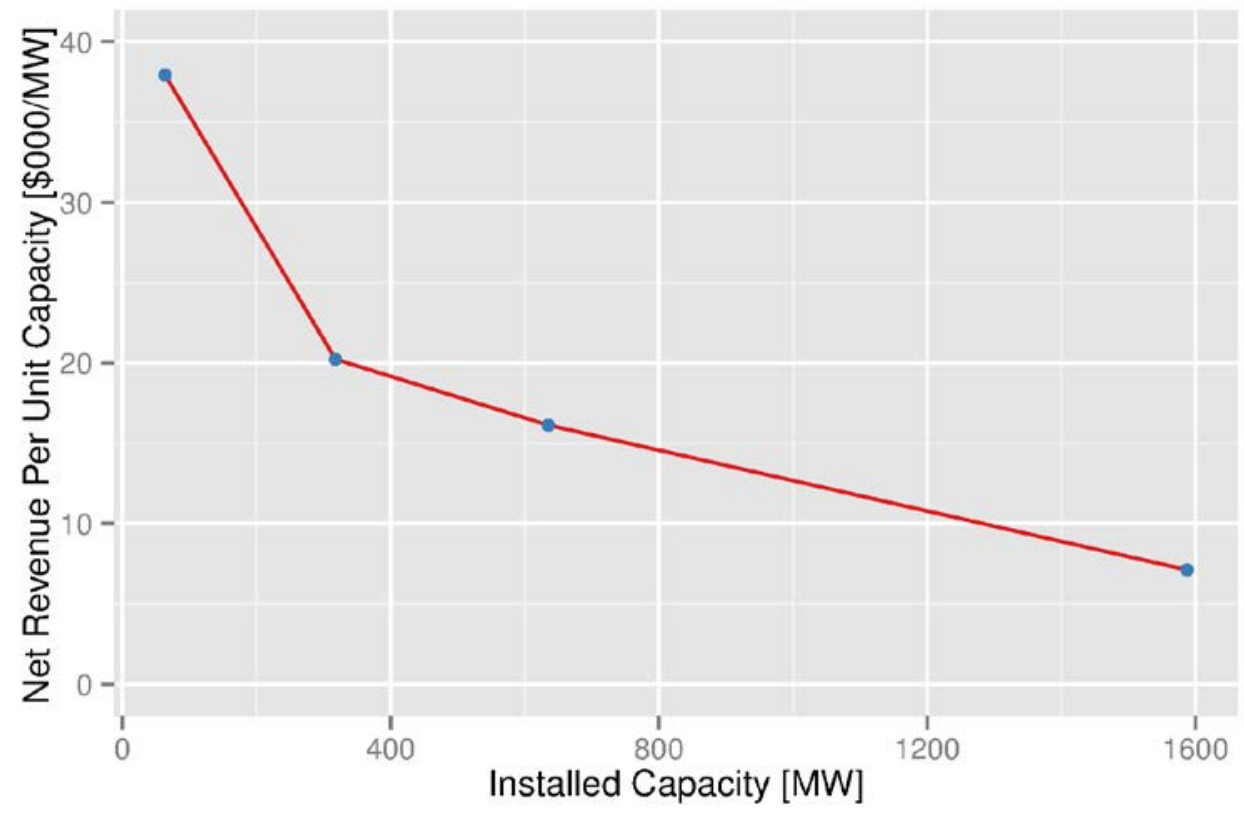

Figure 17. Annual DR revenue per MW of installed DR capacity

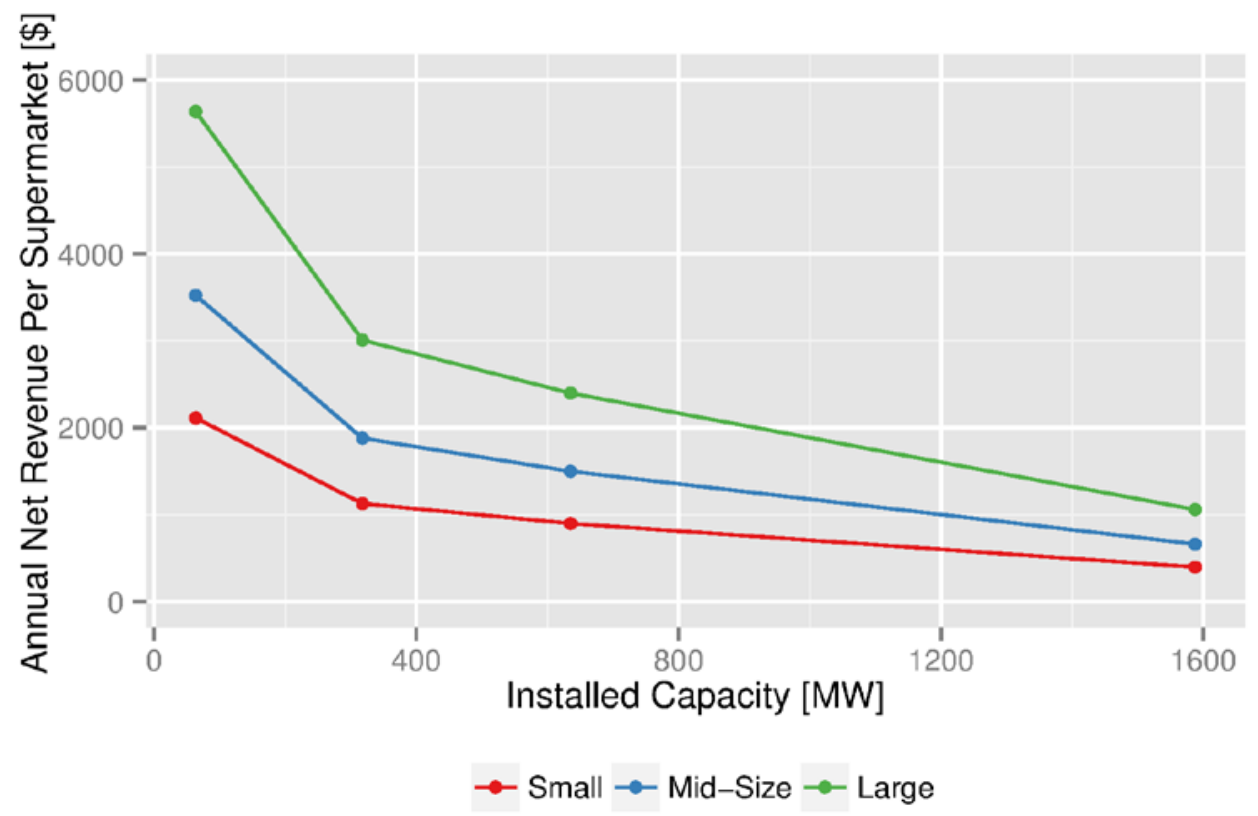

Figure 18. Annual net revenue per participating supermarket 
Figure 19 illustrates the change in the generation dispatch at different levels of DR as compared to the real-time system dispatch without DR, where each column represents one of the DR sensitivity studies, e.g. x5DR is the case with five times the base DR capacity. Demand response is seen to displace generation from less efficient gas combustion turbines (CT), while increasing generation from the more efficient but less flexible combined cycle (CC) gas generators and coal (similar to the findings of [28]). Additionally, DR can be seen to support greater levels of generation from renewable resources, including wind, solar PV, and dispatchable hydropower generation.

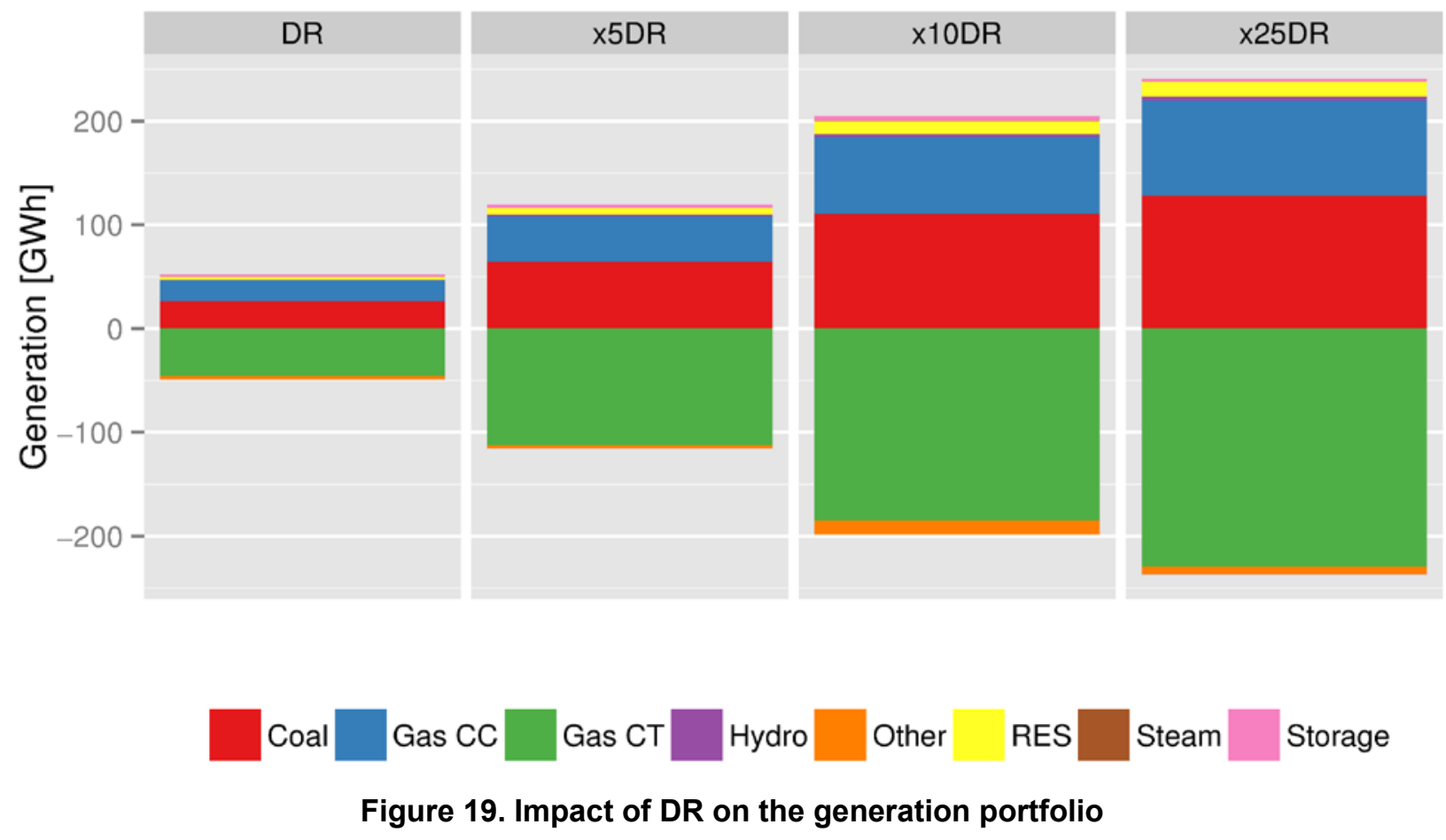

Figure 20 illustrates the price duration curve for each of the levels of DR capacity considered and the case without DR. It is difficult to identify the base case price curve, as it overlaps with that of many of the DR sensitivities for many hours. Between intervals 10,000 and 20,000, the lowest line (in red) is the price duration curve without DR. The introduction of DR increases the price and extends the number of hours during which the price is high, as can be seen by the shift to the right between intervals 25,000 and 30,000. Despite the visible impact on lower priced hours, there is a very limited impact on peak prices. This is an unexpected result, as conventional storage typically mitigates price fluctuations by reducing peak prices and increasing the price during lower priced hours [28]. The impact of this change in the price duration curve can be seen in Figure 17 where the marginal revenue decreases at higher penetrations of DR. This can be attributed to the higher prices during relatively low priced hours, which increases the cost of recovering energy (which usually occurs during low price hours) and consequently reduces the net revenue. 


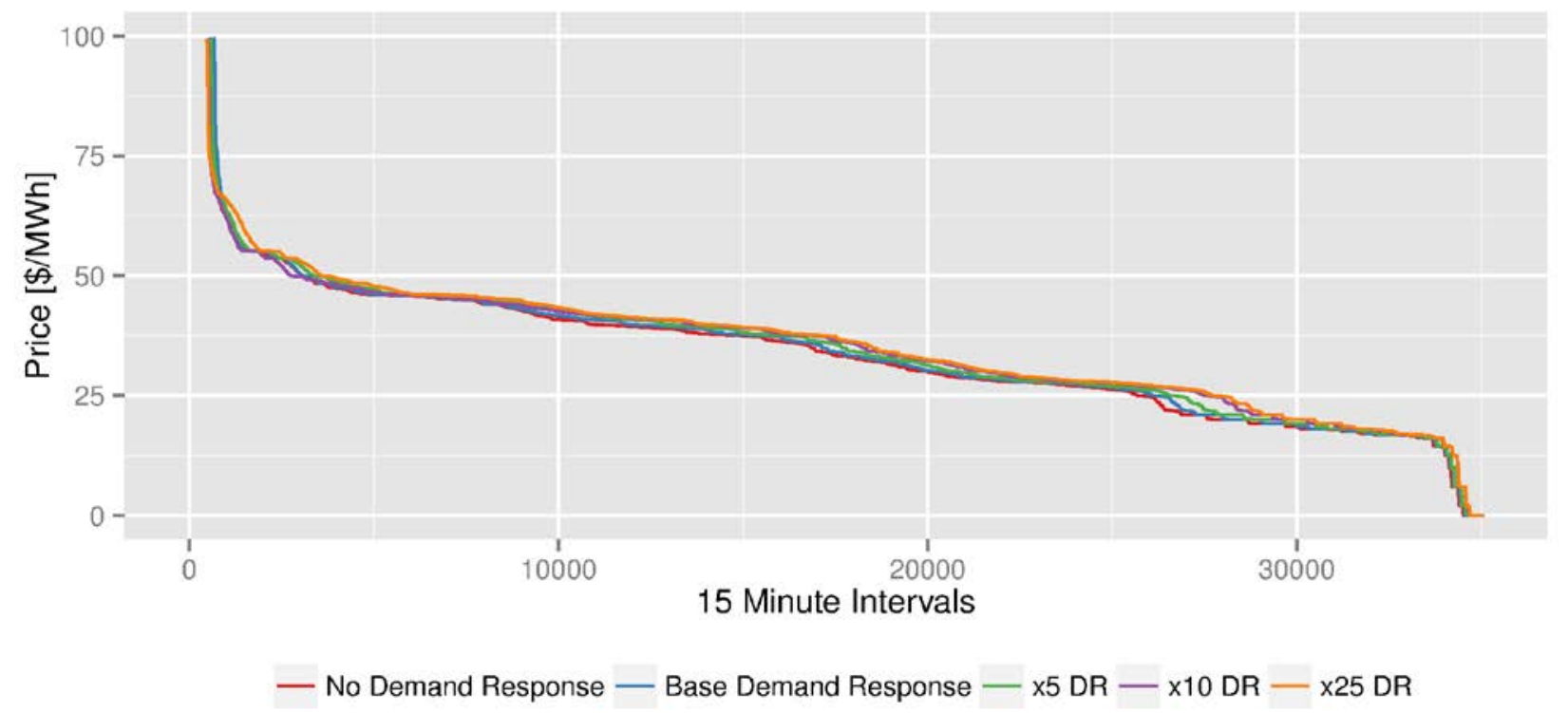

Figure 20. Price duration curves at different levels of installed DR capacity

\subsection{Renewable Energy Sensitivities}

Further sensitivity studies investigate the impact of larger penetrations of renewable resources on the power system with the base DR resource (63.5MW). Figure 21 demonstrates that the value of DR increases with increasing levels of renewable generation, however, the rate of increase slows substantially upon reaching moderate levels of penetration (around 35-45\%) and appears to saturate. This result differs somewhat from the findings of [28], which found a more constant increase in the value of conventional energy storage with increased renewable generation, but does not seem unreasonable based on a) that earlier study also found a deceleration in value in the high $(2 \mathrm{x})$ gas price case and $\mathrm{b})$ the studies offer energy storage into different markets (i.e. day-ahead versus 15-minute markets). At high penetrations of renewables and with the dayahead unit commitment already in place, it is possible for the system to operate primarily on renewable and already-committed base load generators for long periods of time, a situation in which the short-term energy storage provided by the DR resource becomes less valuable. Longer-term storage solutions could still be of interest in this particular situation, and it could be worthwhile in future work to explore initially scheduling DR alongside the day-ahead unit commitment decisions. This scheduling framework may provide greater system value than scheduling solely in real-time, particularly in high renewable penetration scenarios. 


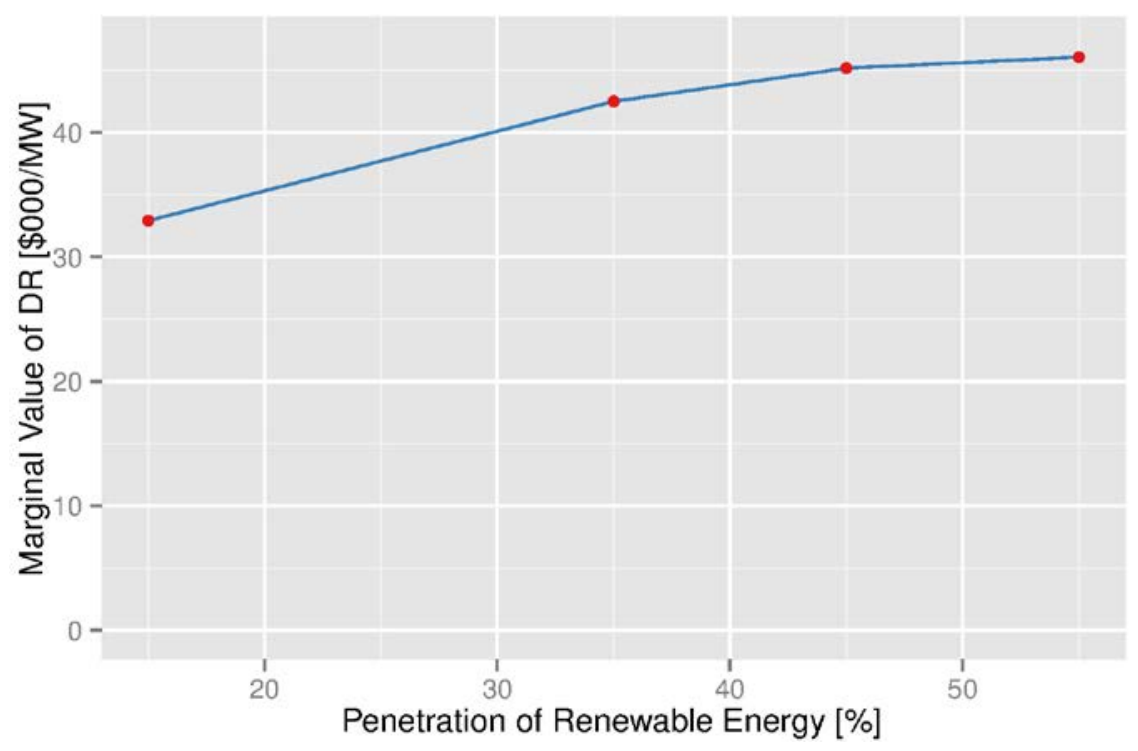

Figure 21. Annual value of DR per MW of installed capacity with increasing penetrations of renewable energy

Figure 22 illustrates the real-time dispatch curve for January 2020, where it can be seen that generation is primarily comprised of renewables and coal. As coal is an inflexible resource, it is expensive to adjust away from its day-ahead dispatch points, so given the choice of displacing coal or renewables, DR will not be dispatched unless the forecast error for renewables is negative and additional generation is required. Figure 23 shows the curtailed renewable generation during this time (January 2020), and clearly demonstrates that the forecast error of renewables is not generally negative. Overall, there is a long-term excess of generation and consequently no need for DR. Offering the DR in the day-ahead market or adjusting the renewables mix toward more solar and less wind could possibly mitigate this situation for this system and this level of renewable generation; however, for most systems we would expect there to be some renewable generation threshold beyond which the main problem is week- or season-long over-supply of (renewable) generation, as depicted in the figures.

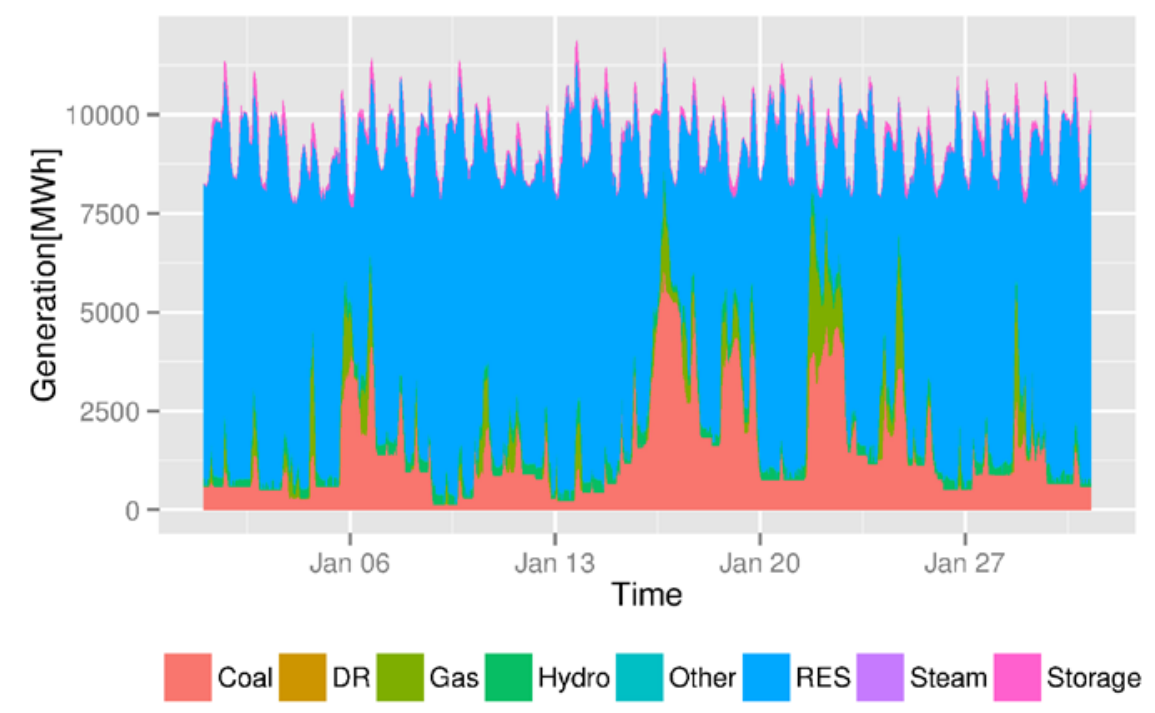

Figure 22. Real-time system dispatch for January 2020 


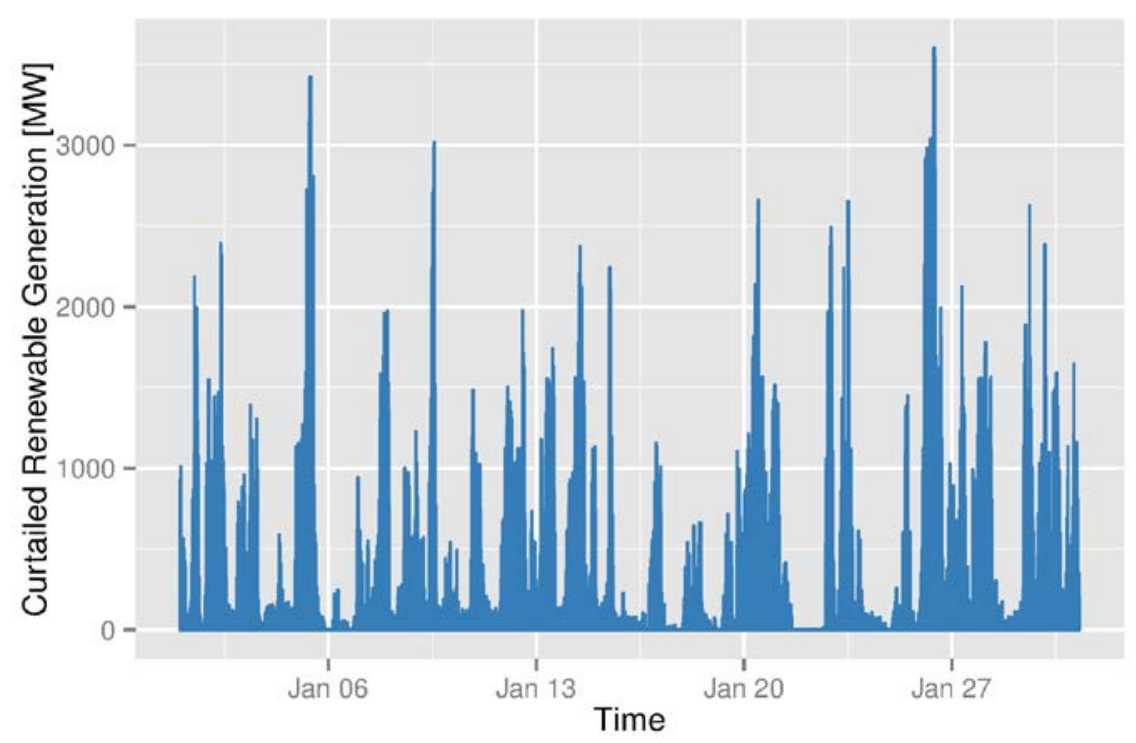

Figure 23. Curtailed renewable generation during January 2020

Figure 24 illustrates the amount of load reduction provided by the DR resource for each of the considered scenarios for renewable energy source (RES) penetration. A significant difference can be observed between the highest-penetration scenario and all other scenarios, particularly during the earlier and latter parts of the year. These periods are also the periods with the greatest amount of generation from renewables. This indicates that the short-term DR modeled here is not as valuable to the system during periods of very high generation from variable renewables, at which times a longer-term source of storage would be more beneficial.

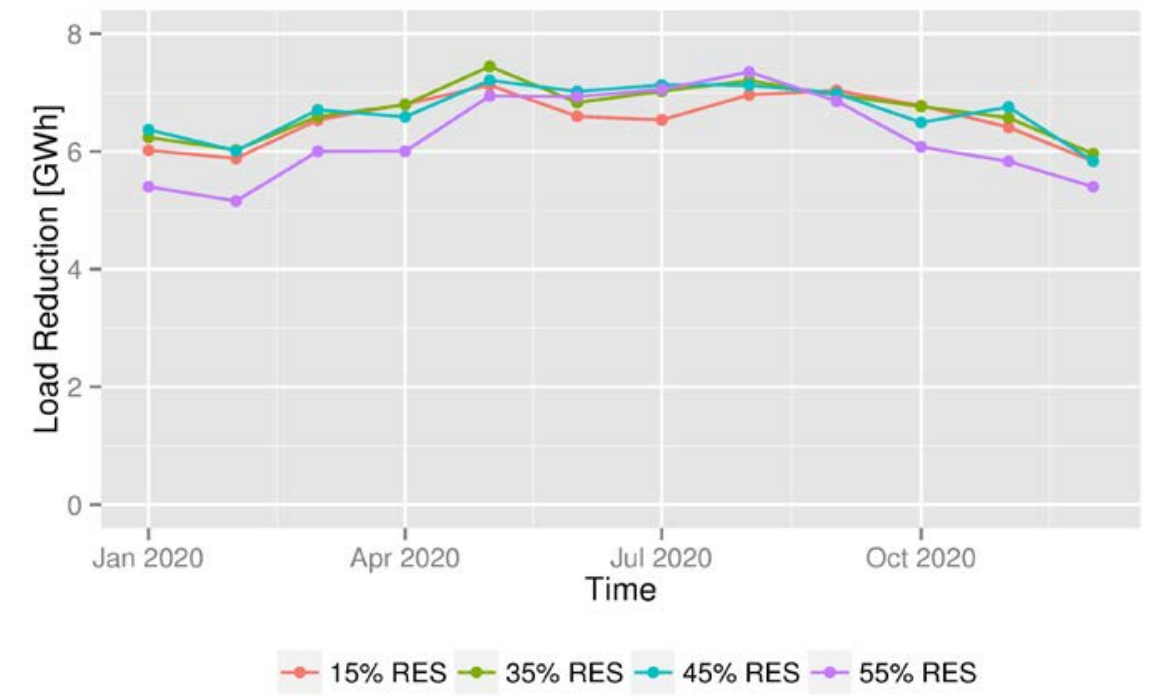

Figure 24. Monthly DR load reduction for each renewable energy penetration scenario 
Figure 25 illustrates the impact of DR on the generation portfolio with increasing penetrations of renewable generation. It can be seen that gas $\mathrm{CT}$ is consistently displaced across all scenarios, while DR supports increased generation from renewable generation sources as the penetration of renewables increases. At lower penetrations of renewables it appears that DR may induce increased emissions due to its support of additional coal generation. At higher penetrations of renewables, this trend is mitigated as DR begins to reduce renewable curtailments more than it supports generation from coal plants.
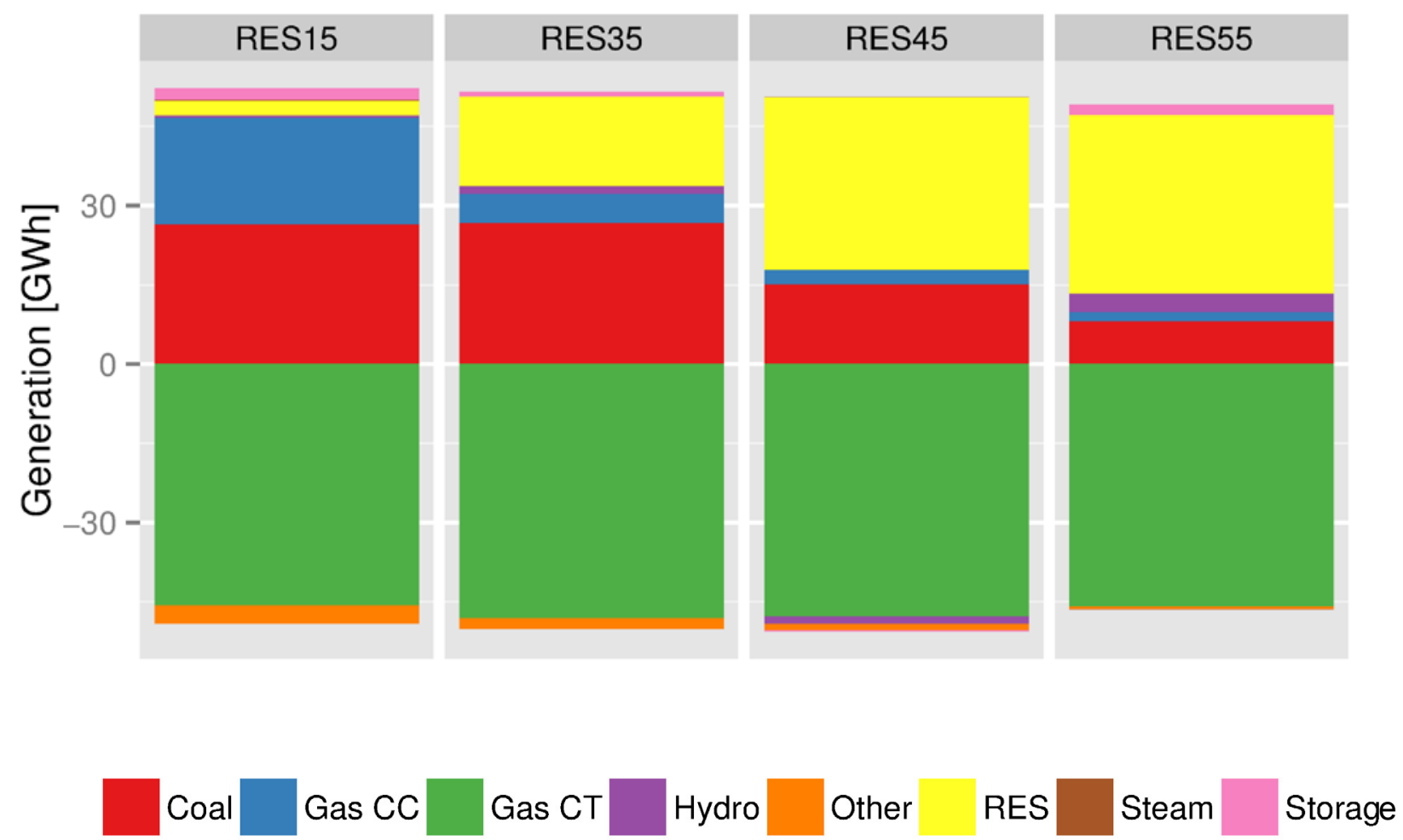

Figure 25. Impact of DR on generation with increasing penetrations of renewable generation $[15 \%, 35 \%, 45 \%$ and $55 \%]$

Figures 26, 27, and 28 demonstrate the impact of increased penetrations of renewables on avoided renewables curtailment, the value of DR per supermarket, and the net revenue per supermarket respectively. In all cases, the increase in renewables improves the case for DR until the penetration of renewables exceeds $45 \%$, at which point the benefit of DR exhibits no further significant increase. 


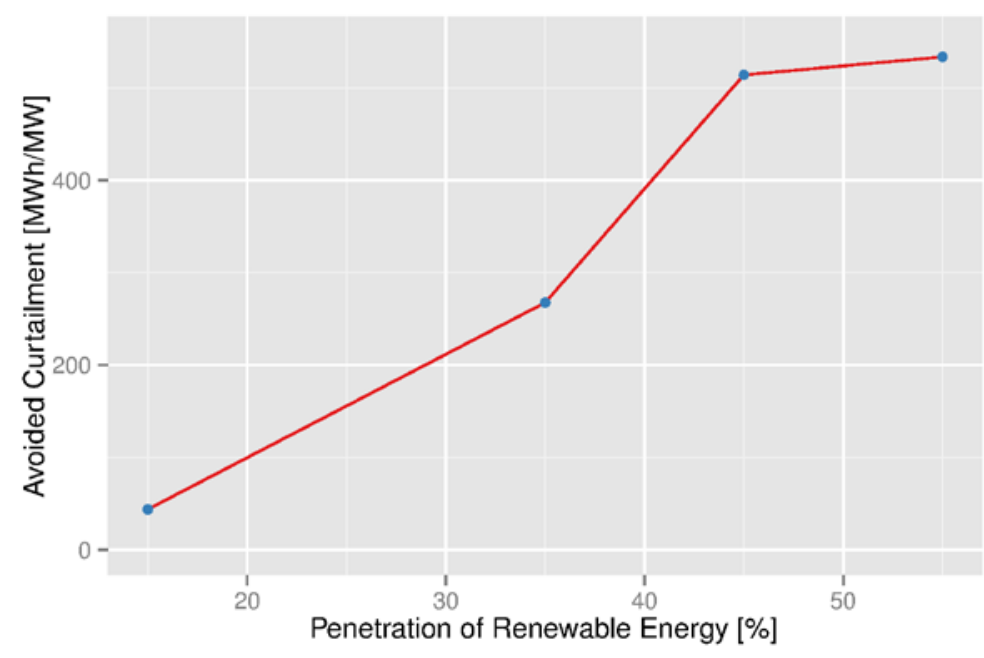

Figure 26. Annual avoided curtailment of renewable generation per MW of enabled DR capacity with increasing penetration of renewable energy

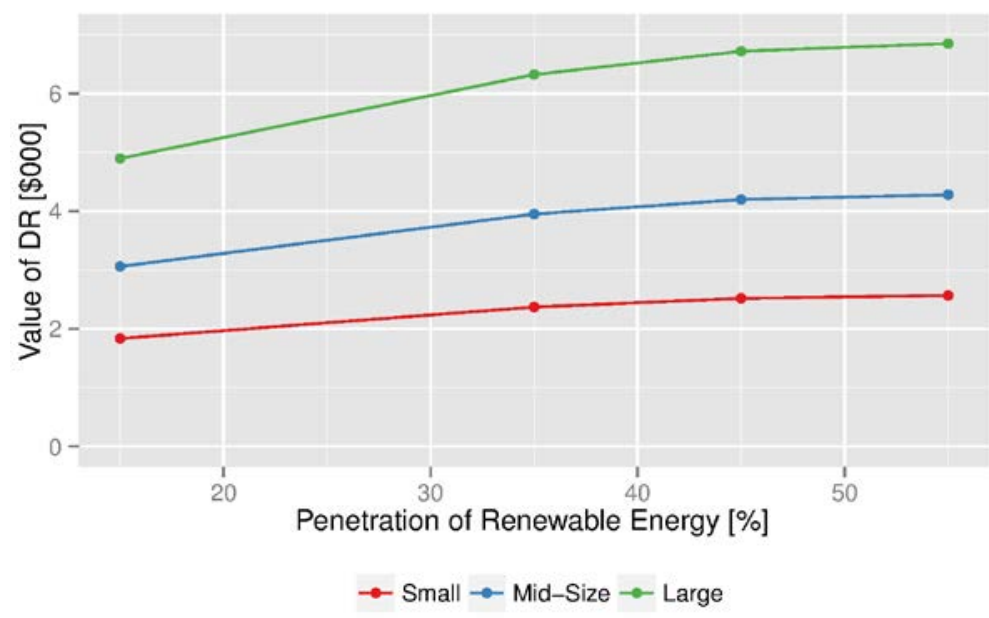

Figure 27. Annual value of DR per participating supermarket with increasing penetrations of renewable energy

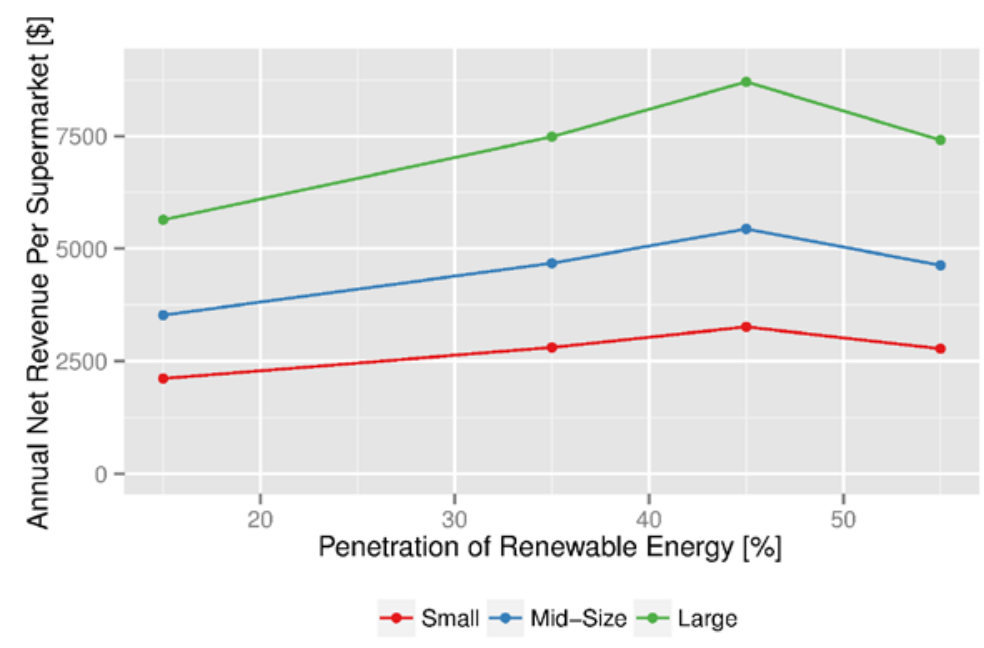

Figure 28. Annual net revenue per supermarket with increasing penetrations of renewable energy 
The trend exhibited by the net revenue of DR with increasing penetrations of renewables is seen to reverse at the highest penetration of renewables considered in these sensitivity studies. This contrasts with the trend of the value of DR, which appears to saturate but not reverse. At increasing penetrations of renewables, peak prices are suppressed, and there is higher incidence of zero-price hours, as shown by the price duration curves in Figure 29. This would indicate that DR could generate greater revenue due to the opportunity to recover energy from the system at no cost. The impact of a greater number of zero-price hours can be seen in Figure 30 where the cost of energy recovery decreases continually with increasing penetrations of renewables. However, as the DR resource is dispatched less frequently (as shown in Figure 24), it has less opportunity to generate revenue through the sale of power. Thus, despite the ability to recover more energy for free, the overall revenue achieved is reduced.

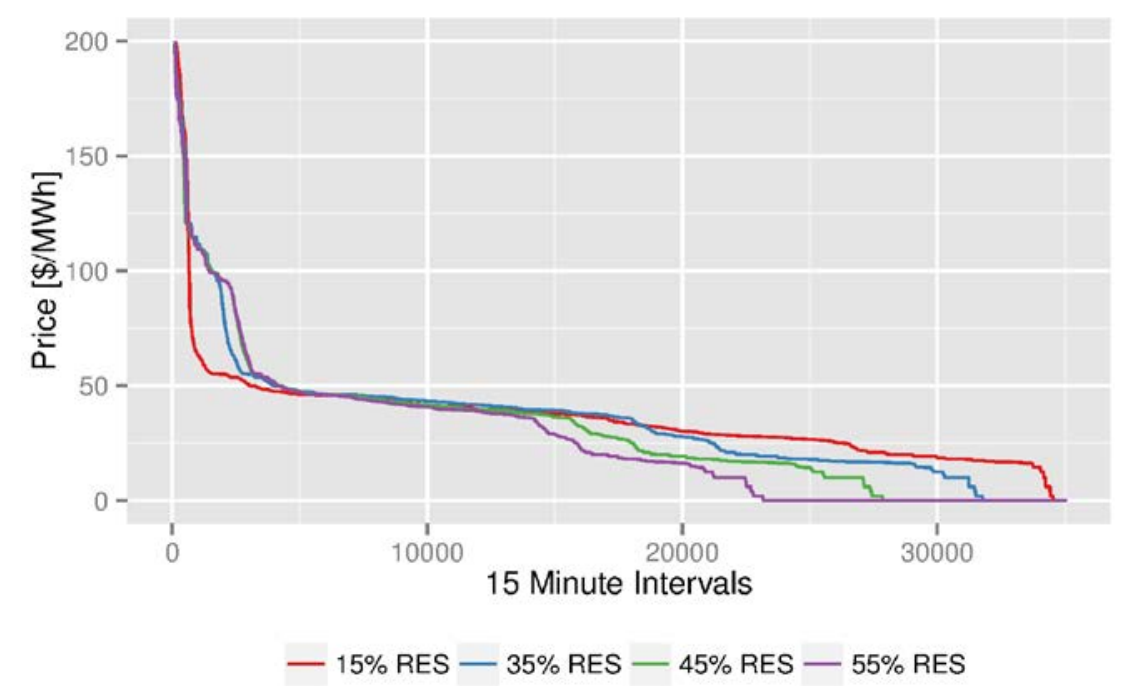

Figure 29. Price duration curve with increasing penetration of renewable energy and the base case DR resource

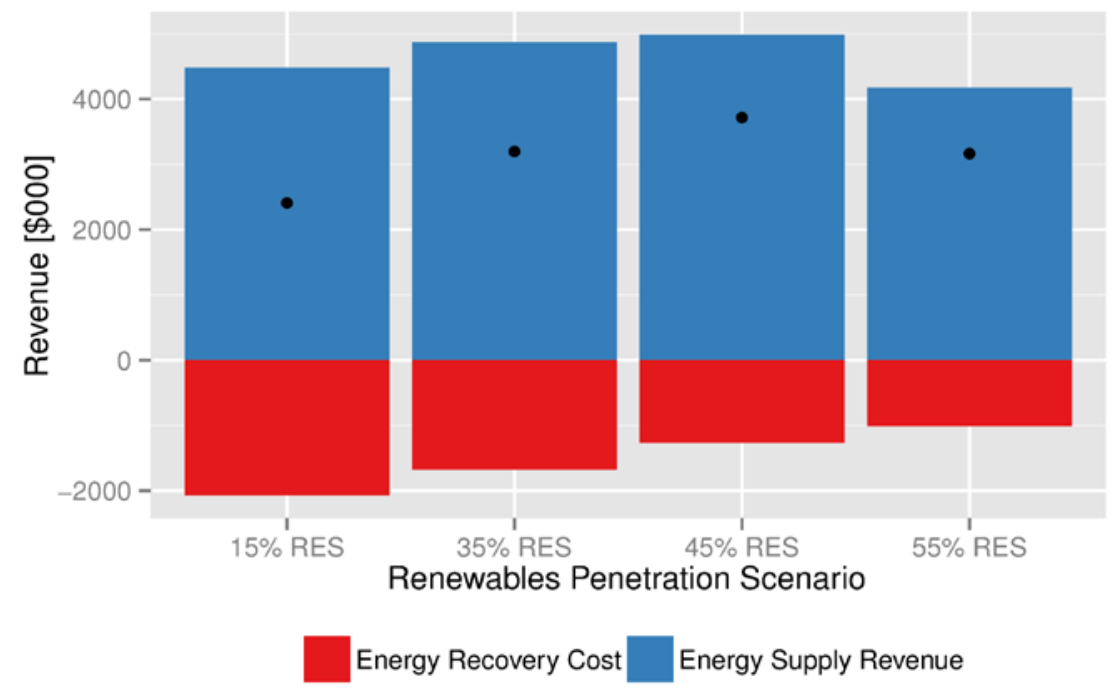

Figure 30. Breakdown of annual DR revenue for each scenario of renewables penetration considered. Net revenue is indicated by the black dots. 


\section{Conclusions}

A methodological framework for the modeling of load-shifting DR using a bottom-up approach has been developed in this work. The modeling methodology is generic and can be applied to a range of thermal-electric loads that are suitable for the provision of load-shifting DR. The model is capable of incorporating the dependency of the resource flexibility on external factors such as ambient temperature and building occupancy, though further work is necessary to fully represent diurnal variations. The methodology developed differs from existing bottom-up DR modeling approaches in that the resulting model is suitable for inclusion in large-scale power system studies of long duration. This facilitates an assessment of the power system operational cost reductions offered by DR over a year.

The modeling methodology is demonstrated using the example of supermarket refrigeration DR resource adjusting compressor power consumption. The flexibility and dynamics of refrigeration are characterized by a saturation curve that relates change in power to the amount of time for which the change can be sustained. Multiple storage configurations, distinguished by different saturation times, are offered for dispatch in the power system as implemented in a commercial production cost modeling tool. The impact of the daily average ambient temperature on the flexibility offered by refrigeration is also characterized and incorporated into the model.

A model representing the load-shifting DR of the population of supermarkets in Colorado is implemented in a production cost model of a test system representing the power system of Colorado. This DR resource is found to have a value of $\$ 32.85 / \mathrm{kW}$-year when it provides an energy service in a 15-minute, real-time market. This value corresponds to the production costs savings achieved by implementing DR and are primarily due to the displacement of gas-fired combustion turbine (CT) plants and through avoided curtailment of renewable generation. The capacity of the population of supermarkets modeled is very small, representing a maximum of $0.24 \%$ of the generation capacity on the system. Consequently, the absolute value it offers per year is very low, at $\$ 2.089$ million, or $\$ 4,890$ for each large supermarket providing DR.

Sensitivity studies revealed the per-unit value of DR decreases as the capacity of the resource increases. The net revenue accrued per supermarket is also found to decrease as the penetration of DR on the system increases. This indicates that the business case for supermarkets or other DR resources to provide DR weakens as the resource grows. It should be noted that prices on the test system are largely driven by fuel costs, and thus the revenue generated by DR is sensitive to the portfolio of generators on the system and their fuel costs. On systems with higher fuel cost differentials and other price components such as carbon costs, it is possible that DR could generate greater revenue.

The framework developed in this work is applicable to a range of flexible loads capable of providing load-shifting DR. An important continuation of this work is an extension of the modelling framework to incorporate DR participation in other power system markets such as capacity and ancillary services. Future work should also consider the application of this methodology to a portfolio of suitable loads, so that a full integration study on load-shifting DR can be conducted. Other key areas of interest for the continuation of this research agenda include the potential synergies between complementary resources such as heating and cooling and the impact of diurnal variations in flexibility on the value of DR. 


\section{References}

[1] GE Energy and National Renewable Energy Laboratory, Western Wind and Solar Integration Study: Executive Summary (Golden, CO: National Renewable Energy Laboratory, 2010).

[2] EnerNex Corporation. 2011. "Eastern Wind Integration and Transmission Study (EWITS) (Revised)." Subcontract Report NREL/SR-5500-47078. Golden, Colorado: National Renewable Energy Laboratory. http://www.nrel.gov/docs/fy1 1osti/47078.pdf.

[3] Nolan, Sheila, Mark O'Malley, Marissa Hummon, Sila Kiliccote, and Ookie Ma, "A Methodology for Estimating the Capacity Value of Demand Response," IEEE PES General Meeting, (2014): 1-5.

[4] Hummon, Marissa, David Palchak, Paul Denholm, Jennie Jorgenson, Daniel J. Olsen, Sila Kiliccote, Nance Matson, Michael Sohn, Cody Rose, Junqiao Dudley, Sasank Goli, and Ookie Ma, "Grid Integration of Aggregated Demand Response, Part 2: Modeling Demand Response in a Production Cost Model," Natl. Renew. Energy Lab. December 2013 (2013).

[5] Olsen, Daniel, Nance Matson, Michael Sohn, Cody Rose, Junqiao Dudley, Sasank Goli, Sila Kiliccote, Marissa Hummon, David Palchak, Paul Denholm, Jennie Jorgenson, and Ookie Ma, "Grid Integration of Aggregated Demand Response, Part 1: Load Availability Profiles and Constraints for the Western Interconnection," 2013.

[6] Zugno, Marco, Juan Miguel Morales, Pierre Pinson, and Henrik Madsen, “A Bilevel Model for Electricity Retailers Participation in a Demand Response Market Environment," Energy Econ. 36 (2012): 182-197.

[7] Lopez, M. A., S. De La Torre, S. Martin, and J. A. Aguado, "Demand-Side Management in Smart Grid Operation Considering Electric Vehicles Load Shifting and Vehicle-toGrid Support," Int. J. Electr. Power Energy Syst. 64 (2015): 689-698.

[8] De Jonghe, Cedric, Benjamin F. Hobbs, and Ronnie Belmans, "Optimal Generation Mix With Short-Term Demand Response and Wind Penetration," IEEE Trans. Power Syst. 27, no. 2 (May 2012): 830-839.

[9] Madaeni, Seyed Hossein and Ramteen Sioshansi, "The Impacts of Stochastic Programming and Demand Response on Wind Integration,” Energy Syst. Sep. 2012 (2012): 1-16.

[10] Bruninx, Kenneth, Dieter Patteeuw, Erik Delarue, Lieve Helsen, and William D'haeseleer, "Short-Term Demand Response of Flexible Electric Heating Systems: The Need for Integrated Simulations," International Conference on the European Energy Market 2012, May (2012): 1-10. 
[11] Mathieu, Johanna L., Tobias Haring, John O. Ledyard, and Goran Andersson, "Residential Demand Response Program Design: Engineering and Economic Perspectives," 2013 10th Int. Conf. Eur. Energy Mark (2013): 1-8.

[12] Roozbehani, Mardavij, Munther A. Dahleh, and Sanjoy K. Mitter, "Volatility of Power Grids Under Real-Time Pricing," IEEE Trans. Power Syst. 27, no. 4 (2012): 1926-1940.

[13] Zerrahn, Alexander and Wolf-Peter Schill, "On the Representation of Demand-Side Management in Power System Models," Energy 84 (2015): 840-845.

[14] Hao, He, Borhan M. Sanandaji, Kameshwar Poolla, and Tyrone L. Vincent, "Aggregate Flexibility of Thermostatically Controlled Loads," IEEE Trans. Power Syst. 30, no. 1 (2015): 189-198.

[15] Marzooghi, Hesamoddin, Gregor Verbic, and David J. Hill, "Aggregated Demand Response Modelling for Future Grid Scenarios," ArXiv - Submitt. to IEEE Trans. Power Syst. (2015): 1-8.

[16] Koch, Stephan, Johanna L. Mathieu, and Duncan S. Callaway, "Modeling and Control of Aggregated Heterogeneous Thermostatically Controlled Loads for Ancillary Services," 17th Power Systems Computation Conference (2011).

[17] Ma, Zhongjing, Duncan Callaway, and Ian Hiskens, "Decentralized Charging Control for Large Populations of Plug-in Electric Vehicles," IEEE Conference on Decision and Control (2010): 206-212.

[18] O'Connell, Niamh, Henrik Madsen, Pierre Pinson, and Mark O'Malley, "Modelling and Assessment of the Capabilities of a Supermarket Refrigeration System for the Provision of Regulating Power," DTU Compute - 2013 - 24, 2013.

[19] O'Connell, Niamh, Henrik Madsen, Pierre Pinson, Mark O'Malley, and Torben Green, "Regulating Power from Supermarket Refrigeration," Proceedings of the IEEE ISGT Europe 2014 (2014).

[20] Ali, Mubbashir, Antti Alahäivälä, Farhan Malik, Muhammad Humayun, Amir Safdarian, and Matti Lehtonen, "A Market-Oriented Hierarchical Framework for Residential Demand Response," Int. J. Electr. Power Energy Syst., 69 (2015): 257-263.

[21] Borsche, Theodor, Frauke Oldewurtel, and Göran Andersson, "Scenario-Based MPC for Energy Schedule Compliance with Demand Response," 19th World Congress of The International Federation of Automatic Control (2014): 10299-10304.

[22] Zhang, Wei, Karanjit Kalsi, and Jason Fuller, "Aggregate Model for Heterogeneous Thermostatically Controlled Loads with Demand Response," Power Energy (2012): 1-8. 
[23] California Energy Commission and Itron Inc., California Commercial End-Use Survey (CEUS), CEC-400-2006-005, (2006).

[24] Morales-España, Germán, Carlos M. Correa-Posada, and Andres Ramos, "Tight and Compact MIP Formulation of Configuration-Based Combined-Cycle Units," IEEE Trans. Power Syst., PP, no. 99 (2015): 1-10.

[25] “The General Algebraic Modeling System (GAMS)," 2014. [Online]. Available: www.gams.com.

[26] Energy Information Administration, "2003 CBECS Building Characteristics and Consumption and Expenditures for All Buildings ( Including Malls )," Energy Information Administration, 2008. [Online]. Available:

http://www.eia.gov/consumption/commercial/data/2003/\#a1.

[27] United States Census Bureau, "County Business Patterns," 2015. [Online]. Available: http://www.census.gov/econ/cbp/.

[28] Denholm, Paul, Jennie Jorgenson, Marissa Hummon, David Palchak, Brendan Kirby, Ookie Ma, and Mark O. Malley, The Impact of Wind and Solar on the Value of Energy Storage, (Golden, CO: National Renewable Energy Laboratory, 2013).

[29] Hummon, Marissa, Paul Denholm, Jennie Jorgenson, David Palchak, Brendan Kirby, and Ookie Ma, Fundamental Drivers of the Cost and Price of Operating Reserves, (Golden, CO: National Renewable Energy Laboratory, 2013. 